\title{
A non-linear anisotropic hyperelastic formulation for granular materials: comparison with existing models and validation
}

\author{
Angelo Amorosi ${ }^{(1)}$, Fabio Rollo ${ }^{(1)}$, Guy T. Houlsby ${ }^{(2)}$ \\ (1) Sapienza Università di Roma, Department of Structural and Geotechnical Engineering, via Eudossiana 18, 00184 Roma, \\ Italy (phone: +39-06-44585982, e-mail: angelo.amorosi@uniroma1.it). \\ (2) University of Oxford, Department of Engineering Science, Oxford OX1 3PJ, UK
}

* Corresponding Author

Keywords: constitutive modelling, hyperelasticity, anisotropy, small strain shear stiffness 


\begin{abstract}
In this paper the non-linear anisotropic hyperelastic model recently proposed by Houlsby, Amorosi and Rollo [27] is critically analysed and validated against experimental data. The model, here mainly discussed in terms of its strain energy function, is initially compared to existing formulations for the cases of non-linear isotropic, linear anisotropic and the complete non-linear anisotropic formulation. It is observed that the proposed model, which employs very few material parameters, encompasses most, but not all, of the existing anisotropic hyperelastic ones and provides a satisfactory fitting to laboratory experimental data observed on both sands and clays.
\end{abstract}




\section{Introduction}

Soils when subjected to very small stress/strain perturbations exhibit a response that can be considered reversible and modelled by elasticity theory. Experimental observations indicate that the reversible stiffness is characterised by a non-linear dependence on the stress state, often combined with a directional character. This latter can be related to either inherent or stress-induced anisotropy: the first stemming from internal microstructural features, such as particle orientation and void distribution, while the second is directly associated with the directional character of the current stress state [37]. Small strain stiffness anisotropy plays an important role in many geotechnical problems, as demonstrated numerically for example in [16, 20, 40].

Hyperelasticity is a convenient tool to develop reversible models for soils, given its thermodynamic consistency. Several hyperelastic constitutive laws have been proposed in the literature to account for stress dependency, in an either linear or non-linear fashion (e.g. [5, 6, 14, 25, 30, 39, 43]), while others were aimed at incorporating inherent anisotropy via a fabric tensor [3, 34]. Most of these models do not account for both non-linearity and anisotropy at the same time, with the notable exceptions of those proposed by Gajo \& Bigoni [17], Cudny \& Partyka [11] and recently by Xiao et al. [45] and Houlsby et al. [27]. This latter, hereafter referred to as HAR18, is based on the non-linear isotropic formulation proposed by Houlsby et al. [26], extended to include orthotropy via a second order symmetric tensor a, following an approach similar to that proposed by Lodge [34] for linear elasticity.

In HAR18 [27] the mathematical formulation is set out, while no specific indications are provided on the similarities and differences with respect to other models, on the calibration procedure of its parameters or on its predictive capability as estimated from observed experimental data. This paper is aimed at bridging the above gaps, illustrating the model performance for the linear and non-linear isotropic and anisotropic cases, comparing it to existing elasticity models and assessing its capability of reproducing soil behaviour as observed during accurate experiments involving the very small strain regime of both sandy and clayey soils.

In contrast to HAR18 [27], the model is here mainly discussed in terms of its strain energy formulation, as this is a key ingredient of most finite element implementations for the analysis of geotechnical boundary value problems.

In the following the soil mechanics sign convention (compressive positive) is assumed, and all stresses are effective stresses. All tensor and vector quantities are written in boldface (componentfree) form, italic letters are used for vectors. Considering the Cartesian basis $\boldsymbol{e}_{i}, \boldsymbol{e}_{j}, \boldsymbol{e}_{k}, \boldsymbol{e}_{l}$ and two 
second order tensors $\mathbf{a}$ and $\mathbf{b}$ we define the products $\mathbf{a b}=a_{i j} b_{j k} \boldsymbol{e}_{i} \boldsymbol{e}_{k}, \mathbf{a} \otimes \mathbf{b}=a_{i j} b_{k l} \boldsymbol{e}_{i} \boldsymbol{e}_{j} \boldsymbol{e}_{k} \boldsymbol{e}_{l}$ and $\mathbf{a} \underline{\otimes} \mathbf{b}=\frac{1}{2}\left(a_{i k} b_{j l}+a_{i l} b_{j k}\right) \boldsymbol{e}_{i} \boldsymbol{e}_{j} \boldsymbol{e}_{k} \boldsymbol{e}_{l}$. If the two second order tensors $\mathbf{a}$ and $\mathbf{b}$ correspond to the second order identity tensor $\mathbf{I}=\delta_{i j} \boldsymbol{e}_{i} \boldsymbol{e}_{j}$, where $\delta_{i j}$ is the Kronecker delta, then $\mathbf{I} \underline{\otimes} \mathbf{I}=\frac{1}{2}\left(\delta_{i k} \delta_{j l}+\delta_{i l} \delta_{j k}\right) \boldsymbol{e}_{i} \boldsymbol{e}_{j} \boldsymbol{e}_{k} \boldsymbol{e}_{l}=\mathrm{I}^{s y m}$, such that $\mathrm{I}^{s y m}=\boldsymbol{\varepsilon}=\boldsymbol{\varepsilon}^{s y m}=\frac{1}{2}\left(\boldsymbol{\varepsilon}+\boldsymbol{\varepsilon}^{T}\right)$ defines the symmetrised strain. The trace of a second order tensor is $\operatorname{tr} \mathbf{a}=a_{i i}$. The strain tensor $\boldsymbol{\varepsilon}=\frac{1}{3} \operatorname{tr}(\boldsymbol{\varepsilon}) \mathbf{I}+\boldsymbol{\varepsilon}^{\prime}$ and the effective stress $\sigma=\operatorname{tr}(\boldsymbol{\sigma}) \mathbf{I}+\boldsymbol{\sigma}^{\prime}$ are symmetric, with the prime denoting their deviatoric parts. The stress invariants employed here are the mean pressure $p=\frac{1}{3} \operatorname{tr} \sigma=\frac{1}{3} \sigma_{i i}$ and the deviatoric stress $q=\sqrt{\frac{3}{2} \operatorname{tr}\left(\sigma^{\prime}\right)^{2}}=\sqrt{\frac{3}{2} \sigma^{\prime}{ }_{i j} \sigma^{\prime}{ }_{j i}}$ and the strain invariants of the conjugate strain are the volumetric strain $\varepsilon_{v}=\operatorname{tr} \varepsilon=\varepsilon_{i i}$ and the deviatoric strain $\varepsilon_{s}=\sqrt{\frac{2}{3} \operatorname{tr}\left(\varepsilon^{\prime}\right)^{2}}=\sqrt{\frac{2}{3} \varepsilon^{\prime}{ }_{i j} \varepsilon_{j i}^{\prime}}$.

\section{Outline of the model}

For the reasons discussed in detail in HAR18 [27], in the following we define the strain energy function in terms of a "symmetrised" strain $\boldsymbol{\varepsilon}^{\mathrm{sym}}$. In brief this is to ensure that on differentiation twice this leads to a stiffness matrix which respects the minor symmetries. Other authors have adopted a different approach, writing the strain without explicitly symmetrising it, but introducing special rules for differentiation which result in a stiffness matrix with minor symmetries. When comparing models, we use the $\boldsymbol{\varepsilon}^{\text {sym }}$ form for our proposed model, for consistency with HAR18 [27]. However, we simply use $\boldsymbol{\varepsilon}$ when describing models proposed by other authors, for consistency with their original papers.

The elastic strain energy proposed in HAR18 [27] for the case $0 \leq n<1$, where the parameter $n$ controls the non-linear dependence on the stress state, can be written in the most general form as follows:

$$
E(\overline{\boldsymbol{\varepsilon}})=\frac{p_{r}}{k(2-n)}\left[\bar{r}^{(2-n) /(1-n)}-N\right]
$$

where $\bar{r}_{0}^{2}$ is given by: 


$$
\bar{r}_{0}^{2}=[k(1-n) \operatorname{tr} \overline{\boldsymbol{\varepsilon}}-N]^{2}+2 g k(1-n) \operatorname{tr}\left(\overline{\boldsymbol{\varepsilon}}^{\prime s y m^{2}}\right)
$$

In eq. (1) $n, k$ and $g$ are dimensionless parameters and $p_{r}$ is a reference stress, typically corresponding to the atmospheric pressure, while the switch parameter $N$ allows the reference stress point for zero elastic strain on the isotropic axis to be switched from $\sigma=0(N=0)$ to the mean effective stress equal to $p_{r}$, that is $\sigma=\mathbf{I} p_{r}(N=1)$. Eq. (1) holds for $0 \leq n<1$, and thus encompasses all the possible cases except that of stiffness linearly dependent on stress $(n=1)$.

The equivalent strain tensor is defined as $\bar{\varepsilon}=\mathbf{a} \mathbf{a}$ where $\mathbf{a}$ is a symmetric second order fabric tensor, which accounts for the inherent anisotropy. The existence of a strain energy potential and a complementary energy potential allows the entire elastic response to be derived in a thermodynamically acceptable way. The stress tensor is obtained based on the definition of the equivalent stress tensor $\overline{\boldsymbol{\sigma}}$ reported in HAR18 [27]:

$$
\boldsymbol{\sigma}=\mathbf{a} \overline{\boldsymbol{\sigma}} \mathbf{a}=\mathbf{a} \frac{\partial E(\overline{\boldsymbol{\varepsilon}})}{\partial \overline{\boldsymbol{\varepsilon}}} \mathbf{a}
$$

while the stiffness tensor is expressed by further differentiation:

$$
\mathbf{D}=(\mathbf{a} \underline{\bar{\otimes}} \mathbf{a}) \frac{\partial^{2} E(\overline{\boldsymbol{\varepsilon}})}{\partial \overline{\mathbf{\varepsilon}} \otimes \partial \overline{\boldsymbol{\varepsilon}}}(\mathbf{a} \underline{\bar{\otimes}} \mathbf{a})
$$

In the following sections the model performance is evaluated and compared to that of other formulations. For this purpose, it is convenient to rewrite eq. (2) accounting for the definition of the deviatoric part of the equivalent strain tensor $\bar{\varepsilon}^{\prime}=\bar{\varepsilon}-1 / 3 \operatorname{tr}(\overline{\boldsymbol{\varepsilon}}) \mathbf{I}$ :

$$
\bar{r}_{0}^{2}=k(1-n)\left\{\left[k(1-n)-\frac{2}{3} g\right] \operatorname{tr}^{2}(\overline{\boldsymbol{\varepsilon}})+2 g \operatorname{tr}\left(\overline{\boldsymbol{\varepsilon}}^{\text {sym }}{ }^{2}\right)\right\}+N[N-2 k(1-n) \operatorname{tr}(\overline{\boldsymbol{\varepsilon}})]
$$

Furthermore, for the sake of simplicity we limit here the discussion to the case $N=0$, thus assuming the reference point for zero strain at zero stress, as is conventional in most elastic models. Substituting eq. (5) in eq. (1), gives the strain energy in the form:

$$
E(\overline{\boldsymbol{\varepsilon}})=\frac{p_{r}}{k(2-n)}\{k(1-n)\}^{\frac{2-n}{2-2 n}}\left\{\left[k(1-n)-\frac{2}{3} g\right] \operatorname{tr}^{2}(\overline{\boldsymbol{\varepsilon}})+2 g \operatorname{tr}\left(\overline{\boldsymbol{\varepsilon}}^{s y m^{2}}\right)\right\}^{\frac{2-n}{2-2 n}}
$$

The above will be our reference potential throughout this paper.

\section{Isotropic elasticity: linear and non-linear formulation}

The isotropic case is obtained by assuming $\mathbf{a}=\mathbf{I}$, thus $\bar{\varepsilon}=\varepsilon$. 
For the linear case, $n=0$, the elastic stiffness tensor can be written:

$$
\mathbf{D}=p_{r}\left(k-\frac{2}{3} g\right) \mathbf{I} \otimes \mathbf{I}+2 p_{r} g(\mathbf{I} \otimes \bar{\otimes} \mathbf{I})
$$

In the non-linear case, $0 \leq n<1$, the stiffness can be expressed as:

$$
\begin{aligned}
\mathbf{D} & =p_{r}\left[k(1-n)-\frac{2}{3} g\right]\left\{k r_{0}^{\frac{3 n-2}{1-n}} n\left[k(1-n)-\frac{2}{3} g\right] \operatorname{tr}^{2}(\boldsymbol{\varepsilon})+r_{0}^{\frac{n}{1-n}}\right\} \mathbf{I} \otimes \mathbf{I}+ \\
& +4 p_{r} k r_{0}^{\frac{3 n-2}{1-n}} n g^{2}\left(\boldsymbol{\varepsilon}^{s y m} \otimes \boldsymbol{\varepsilon}^{s y m}\right)+2 p_{r} k r_{0}^{\frac{3 n-2}{1-n}} n g\left[k(1-n)-\frac{2}{3} g\right] \operatorname{tr}(\boldsymbol{\varepsilon})\left(\boldsymbol{\varepsilon}^{s y m} \otimes \mathbf{I}+\mathbf{I} \otimes \boldsymbol{\varepsilon}^{s y m}\right)+ \\
& +2 p_{r} r_{0}^{\frac{n}{1-n}} g(\mathbf{I} \otimes \mathbf{~} \mathbf{I})
\end{aligned}
$$

and under triaxial (i.e. axisymmetric) conditions this simplifies into:

$$
\left(\begin{array}{l}
\delta p \\
\delta q
\end{array}\right)=\left[\begin{array}{cc}
\frac{\partial^{2} E}{\partial \varepsilon_{v}{ }^{2}} & \frac{\partial^{2} E}{\partial \varepsilon_{s} \partial \varepsilon_{v}} \\
\frac{\partial^{2} E}{\partial \varepsilon_{s} \partial \varepsilon_{v}} & \frac{\partial^{2} E}{\partial \varepsilon_{s}{ }^{2}}
\end{array}\right]\left(\begin{array}{l}
\delta \varepsilon_{v} \\
\delta \varepsilon_{s}
\end{array}\right)=\left[\begin{array}{ll}
D_{11} & D_{12} \\
D_{21} & D_{22}
\end{array}\right]\left(\begin{array}{l}
\delta \varepsilon_{v} \\
\delta \varepsilon_{s}
\end{array}\right)
$$

where:

$$
\begin{aligned}
& D_{11}=p_{r} k^{2} r_{0}^{\frac{3 n-2}{1-n}}(1-n)^{2}\left(k \varepsilon_{v}^{2}+3 g \varepsilon_{s}^{2}\right) \\
& D_{12}=D_{21}=p_{r} k^{2}(1-n) r_{0}^{\frac{3 n-2}{1-n}} 3 g n \varepsilon_{v} \varepsilon_{s} \\
& D_{22}=p_{r} k r_{0}^{\frac{3 n-2}{1-n}} 3 g\left(k(1-n)^{2} \varepsilon_{v}^{2}+3 g \varepsilon_{s}^{2}\right)
\end{aligned}
$$

Once calibrated, usually under triaxial stress states, the model can be used to explore the reversible response for more general loading conditions (non-isotropic, non-triaxial states).

\subsection{Stress/strain induced anisotropy}

A key feature of eq. (6) in the isotropic case $(\mathbf{a}=\mathbf{I}$, thus $\bar{\varepsilon}=\varepsilon)$ is that it reproduces the experimentally observed dependence of soil stiffness on the current stress state within a non-linear hyperelastic formulation. An interesting side effect is the resulting volumetric-deviatoric coupling, which naturally stems from the energy-based formulation when the material is subjected to nonisotropic stress/strain states. This can be easily verified, under simplified triaxial conditions, by the presence of the off-diagonal terms $D_{12}=D_{21}$ in eq. (9) for $\varepsilon_{\mathrm{s}}>0$.

To highlight this feature, simulated tests were performed using the set of parameters in Table 1, under either constant volumetric (i.e. undrained) or constant deviatoric strain conditions. Fig. 1 illustrates the results in terms of volumetric and deviatoric strain contours plotted in $p-q$ space. It 
confirms that, in contrast to the behaviour of a linear isotropic material, a purely volumetric strain path does not correspond to a constant deviatoric stress path and also a purely distortional strain path does not lead to a constant $p$ stress path.

In granular materials different possible sources of volumetric-deviatoric coupling exist, the most obvious one being related to irreversible behaviour (i.e. plasticity): it is thus worth commenting that in all of the above, by definition, no plastic deformation occurs.

The volumetric-deviatoric coupling has often been termed in the literature as "stress induced anisotropy". This latter should not be confused with inherent anisotropy. The former essentially accounts for directional properties that are induced solely by the current anisotropic stress/strain state, as recently highlighted in Kuhn et al. [31] and Yang et al. [46] from a micromechanical perspective, while the latter is related to pre-existing features of the soil, possibly stemming from its internal structure, such as the prevailing orientation of the particles. The isotropic version of the model, as obtained for $\mathbf{a}=\mathbf{I}$, is formulated purely in terms of invariants of the strain tensor, i.e. no fabric-related directional information is included: as such, the stress-induced anisotropy reproduced by the model is not related to any underlying anisotropic structural character, but rather to the current orientation of the principal stress directions and the relative values of the corresponding stiffness tensor components. This can easily be detected by unloading the material from any initial anisotropic state back to an isotropic stress/strain condition: in this case the hyperelastic formulation will obviously return an isotropic response.

Stress induced anisotropy can be further highlighted by simulating tests under more general stress/strain conditions, using eq. (8), to illustrate the modification of the stiffness matrix for different states of strain. The parameters of the model are again those in Table 1. Under an isotropic strain state, the terms $D_{1111}, D_{2222}$ and $D_{3333}$ are all equal as shown in Table 2, and could be expressed in terms of a current uniquely defined Young's modulus and Poisson's ratio, or equivalently bulk and shear moduli.

Increasing the strain component $\varepsilon_{11}$ leads both to an overall modification of the stiffness matrix, because of non-linearity, and to a stiffer component $D_{1111}$, as compared to $D_{2222}$ and $D_{3333}$, as shown in Table 3.

When a shear strain component $\varepsilon_{12}$ is added to a isotropic state, the off-diagonal terms $D_{1112}=$ $D_{1211}$ are activated and the corresponding shear stiffness term $D_{1212}$ is larger than those on the other planes $\left(D_{1313}=D_{2323}\right)$, see Table 4 . 


\section{Linear anisotropic elasticity}

The anisotropic behaviour of soils is a macroscopic manifestation of their internal microstructure, such as particle orientation, often combined with the distribution of voids and, in some cases, of fissures or cracks. Experimental evidence can be found, for example, in [38], where observations of the microstructural orientation characteristics of a clayey soil is discussed in relation to its anisotropic elastic response, observed at the macroscopic level by propagating shear waves polarised along different planes. From a mathematical point of view, a possible strategy to link these microstructural characteristics to the macroscopic mechanical behaviour is to introduce a symmetric second order fabric tensor that can express all scalar and directional information pertaining to the anisotropy of the material. The use of a second order tensor restricts the material symmetry to orthotropy if its three eigenvalues are distinct and, as special cases, transverse isotropy if two of them are identical and isotropy if the tensor is proportional to the identity tensor. The description of other material symmetries would require the introduction of higher order fabric tensors, but this is beyond the scope of this paper.

As briefly discussed in HAR18 [27], there are different ways to introduce anisotropy in the reversible behaviour of soils. One is based on the formulation of a free energy potential which no longer depends solely on the strain tensor, as in the previous section, but is enriched by dependence on the fabric tensor. The two can be combined consistently by using the representation theorems for scalar valued functions $[4,42,44]$. This approach leads to the most general form for the strain energy potential, in terms of a set of irreducible invariants of the strain and fabric tensors. Within this theoretical framework, Cowin [10] introduced a second order symmetric fabric tensor into the elastic stiffness tensor. In the most general case of orthotropy, nine independent constants have to be defined, which reduce to five and two for transverse isotropy and isotropy, respectively.

Zysset and Curnier [48] considered the directional properties of materials as characterised by an orientation distribution function:

$$
f(\boldsymbol{n})=f+\boldsymbol{n} \mathbf{F} \boldsymbol{n}
$$

where the vector $\boldsymbol{n}$ specifies the internal structural orientation. The microstructural properties of the material are described by a scalar $f$, which is the average of the function, and a traceless second order tensor F. Boehler [4] specifies a list of the corresponding irreducible invariants: 
$\operatorname{tr}(\varepsilon), \operatorname{tr}\left(\varepsilon^{2}\right), \quad \operatorname{tr}\left(\varepsilon^{3}\right)$

$f, \operatorname{tr}\left(\mathbf{F}^{2}\right), \operatorname{tr}\left(\mathbf{F}^{3}\right)$

$\operatorname{tr}(\boldsymbol{\varepsilon} \mathbf{F}), \quad \operatorname{tr}\left(\boldsymbol{\varepsilon}^{2} \mathbf{F}\right), \quad \operatorname{tr}\left(\boldsymbol{\varepsilon} \mathbf{F}^{2}\right), \quad \operatorname{tr}\left((\boldsymbol{\varepsilon} \mathbf{F})^{2}\right)$

leading to a general form of linear anisotropic elasticity, in which the free energy function retains only quadratic terms in $\boldsymbol{\varepsilon}$ :

$$
\begin{aligned}
E & =E(\boldsymbol{\varepsilon}, f, \mathbf{F})=\frac{c_{1}}{2} \operatorname{tr}^{2}(\boldsymbol{\varepsilon})+\frac{c_{2}}{2} \operatorname{tr}\left(\boldsymbol{\varepsilon}^{2}\right)+\frac{c_{3}}{2} \operatorname{tr}^{2}(\boldsymbol{\varepsilon} \mathbf{F})+ \\
& +c_{4} \operatorname{tr}\left(\boldsymbol{\varepsilon}^{2} \mathbf{F}\right)+\frac{c_{5}}{2} \operatorname{tr}^{2}\left(\boldsymbol{\varepsilon} \mathbf{F}^{2}\right)+\frac{c_{6}}{2} \operatorname{tr}\left((\boldsymbol{\varepsilon} \mathbf{F})^{2}\right)+c_{7} \operatorname{tr}(\boldsymbol{\varepsilon}) \operatorname{tr}(\boldsymbol{\varepsilon} \mathbf{F})+ \\
& +c_{8} \operatorname{tr}(\boldsymbol{\varepsilon} \mathbf{F}) \operatorname{tr}\left(\boldsymbol{\varepsilon} \mathbf{F}^{2}\right)+c_{9} \operatorname{tr}(\boldsymbol{\varepsilon}) \operatorname{tr}\left(\boldsymbol{\varepsilon} \mathbf{F}^{2}\right)
\end{aligned}
$$

The stress tensor and the elastic stiffness tensor are obtained by differentiating once and twice the free energy potential with respect to the strain tensor.

Zysset and Curnier [48] proposed a more heuristic way to characterise linear anisotropic elasticity, starting from the classical linear isotropic elastic stiffness tensor:

$$
\mathbf{D}=\lambda \mathbf{I} \otimes \mathbf{I}+2 \mu \mathbf{I} \otimes \underline{\overline{ }} \mathbf{I}
$$

where $\lambda$ and $\mu$ are the two Lamé constants, and substituting the tensor $f \mathbf{I}+\mathbf{F}$ for the identity tensor:

$$
\mathbf{D}=\lambda(f \mathbf{I}+\mathbf{F}) \otimes(f \mathbf{I}+\mathbf{F})+2 \mu(f \mathbf{I}+\mathbf{F}) \stackrel{\otimes}{\otimes}(f \mathbf{I}+\mathbf{F})
$$

This simplification corresponds to certain assumptions about the constants of eq. (13), as follows:

$$
\begin{aligned}
& c_{1}=\lambda f^{2}, \quad c_{2}=2 \mu f^{2}, \quad c_{3}=\lambda \\
& c_{4}=2 \mu f, \quad c_{5}=0, \quad c_{6}=2 \mu \\
& c_{7}=\lambda f, \quad c_{8}=0, \quad c_{9}=0
\end{aligned}
$$

leading to an expression for the free energy function:

$$
\begin{aligned}
E & =E(\boldsymbol{\varepsilon}, f, \mathbf{F})=\frac{\lambda f^{2}}{2} \operatorname{tr}^{2}(\boldsymbol{\varepsilon})+\mu f^{2} \operatorname{tr}\left(\boldsymbol{\varepsilon}^{2}\right)+\frac{\lambda}{2} \operatorname{tr}^{2}(\boldsymbol{\varepsilon} \mathbf{F})+ \\
& +2 \mu f \operatorname{tr}\left(\boldsymbol{\varepsilon}^{2} \mathbf{F}\right)+\mu \operatorname{tr}\left((\boldsymbol{\varepsilon} \mathbf{F})^{2}\right)+\lambda f \operatorname{tr}(\boldsymbol{\varepsilon}) \operatorname{tr}(\boldsymbol{\varepsilon} \mathbf{F})
\end{aligned}
$$

Clearly, when $\mathbf{F}=\mathbf{0}$ isotropic elasticity is recovered.

Following a similar approach, Bigoni and Loret [3] replaced the identity tensor in the elastic stiffness tensor of eq. (14) with the symmetric second order fabric tensor $\mathbf{B}$, assumed to be positive definite:

$$
\mathbf{B}=f \mathbf{I}+\mathbf{F}
$$

leading to the free energy potential: 


$$
E=E(\boldsymbol{\varepsilon}, \mathbf{B})=\frac{\lambda}{2} \operatorname{tr}^{2}(\mathbf{B} \boldsymbol{\varepsilon})+\mu \operatorname{tr}\left[(\mathbf{B} \boldsymbol{\varepsilon})^{2}\right]
$$

and, by differentiating with respect to strain, the stress and stiffness tensors:

$$
\sigma=\lambda \operatorname{tr}(\mathbf{B} \boldsymbol{\varepsilon}) \mathbf{B}+2 \mu \mathbf{B} \boldsymbol{\varepsilon} \mathbf{B}
$$

and

$$
\mathbf{D}=\lambda \mathbf{B} \otimes \mathbf{B}+2 \mu \mathbf{B} \otimes \bar{\otimes} \mathbf{B}
$$

Isotropic elasticity is recovered when $\mathbf{B}=\mathbf{I}$.

If we now refer to our general formulation of eq. (6), it is worth specialising it to the linear anisotropic case $(n=0$ and $\mathbf{a} \neq \mathbf{I})$, giving:

$$
\begin{aligned}
& E(\overline{\boldsymbol{\varepsilon}})=\frac{p_{r}}{2}\left[\left(k-\frac{2}{3} g\right) \operatorname{tr}^{2}(\overline{\boldsymbol{\varepsilon}})+2 g \operatorname{tr}\left(\overline{\boldsymbol{\varepsilon}}^{s y m^{2}}\right)\right]= \\
& =E(\boldsymbol{\varepsilon}, \mathbf{a})=\frac{p_{r}}{2}\left[\left(k-\frac{2}{3} g\right) \operatorname{tr}^{2}(\mathbf{a} \mathbf{a})+2 g \operatorname{tr}\left[\left(\mathbf{a} \boldsymbol{\varepsilon}^{s y m} \mathbf{a}\right)^{2}\right]\right]
\end{aligned}
$$

Comparing the above to eq. (19) it emerges that the two expressions are equivalent, since with $\mathbf{B}=\mathbf{a}^{2}, \operatorname{tr}(\mathbf{B} \boldsymbol{\varepsilon})=\operatorname{tr}(\mathbf{a \varepsilon a})$ and $\operatorname{tr}\left[(\mathbf{B} \boldsymbol{\varepsilon})^{2}\right]=\operatorname{tr}\left[(\mathbf{a} \boldsymbol{\varepsilon} \mathbf{a})^{2}\right]$, further $\lambda=p_{r}\left(k-\frac{2}{3} g\right)$ and $\mu=p_{r} g$.

Differentiating eq. (22) with respect to the strain one obtains the stress and stiffness tensors:

$$
\boldsymbol{\sigma}=p_{r}\left[\left(k-\frac{2}{3} g\right) \operatorname{tr}(\mathbf{a} \boldsymbol{\varepsilon a}) \mathbf{a}^{2}+2 g \mathbf{a}^{2} \boldsymbol{\varepsilon}^{s y m} \mathbf{a}^{2}\right]
$$

and

$$
\mathbf{D}=p_{r}\left(k-\frac{2}{3} g\right) \mathbf{a}^{2} \otimes \mathbf{a}^{2}+2 p_{a} g\left(\mathbf{a}^{2} \otimes \mathbf{a}^{2}\right)
$$

Alternatively, the fabric tensor can be expressed as the sum of its isotropic and deviatoric parts, as in eq. (18). Eq. (22) can then be written:

$$
\begin{aligned}
E(\boldsymbol{\varepsilon}, f, \mathbf{F}) & =\frac{p_{r}}{2}\left(k-\frac{2}{3} g\right)\left\{f^{2} \operatorname{tr}^{2}(\boldsymbol{\varepsilon})+2 f \operatorname{tr}(\boldsymbol{\varepsilon}) \operatorname{tr}\left(\mathbf{F} \boldsymbol{\varepsilon}^{\text {sym }}\right)+\operatorname{tr}^{2}\left(\mathbf{F} \boldsymbol{\varepsilon}^{\text {sym }}\right)\right\}+ \\
& +p_{r} g f^{2} \operatorname{tr}\left(\boldsymbol{\varepsilon}^{s y m^{2}}\right)+2 p_{r} g f \operatorname{tr}\left(\mathbf{F} \boldsymbol{\varepsilon}^{s y m^{2}}\right)+p_{r} g \operatorname{tr}\left[\left(\mathbf{F} \boldsymbol{\varepsilon}^{s y m}\right)^{2}\right]
\end{aligned}
$$

from which one can derive:

$$
\boldsymbol{\sigma}=p_{r}\left[\left(k-\frac{2}{3} g\right) \operatorname{tr}(f \mathbf{I} \boldsymbol{\varepsilon}+\mathbf{F} \boldsymbol{\varepsilon})(f \mathbf{I}+\mathbf{F})+2 g f^{2} \boldsymbol{\varepsilon}^{s y m}+2 g f \boldsymbol{\varepsilon}^{s y m} \mathbf{F}+2 g f \mathbf{F} \boldsymbol{\varepsilon}^{s y m}+2 g \mathbf{F} \boldsymbol{\varepsilon}^{s y m} \mathbf{F}\right]
$$

and: 


$$
\begin{aligned}
\mathbf{D}= & p_{r}\left(k-\frac{2}{3} g\right)\left[f^{2} \mathbf{I} \otimes \mathbf{I}+f(\mathbf{I} \otimes \mathbf{F}+\mathbf{F} \otimes \mathbf{I})+\mathbf{F} \otimes \mathbf{F}\right]+2 p_{r} g f^{2} \mathbf{I} \underline{\otimes} \mathbf{I}+ \\
& +2 p_{r} g f(\mathbf{I} \underline{\otimes} \mathbf{F}+\mathbf{F} \underline{\otimes} \mathbf{I})+2 p_{r} g \mathbf{F} \underline{\otimes} \mathbf{F}
\end{aligned}
$$

\subsection{The role of the constraint on the fabric tensor}

The fabric tensor is intended to describe the directional characteristics of the soil, so it is expedient to normalise it to exclude any purely isotropic measure. In the literature different normalisation rules are proposed; however, no clear indication emerges on which is most appropriate. In this section all the proposed normalisations are examined, highlighting their effects on the fabric tensor, and thus providing further information to guide the user in the appropriate choice.

From a historical perspective, the first introduction of a normalising constraint was that of Lodge [34], who proposed fixing the value to the determinant of the fabric tensor a. A convenient choice is $\operatorname{det}(\mathbf{a})=1$, such that for the isotropic case $(\mathbf{a}=\mathbf{I})$ we recover det $(\mathbf{I})=1$. Bigoni and Loret [3] give a more systematic discussion on the character and constraints of their fabric tensor B. Firstly, for the elastic tensor to be positive definite, $\mathbf{B}$ should also be positive definite, from which it follows that the necessary and sufficient conditions for the positive definiteness of the elastic stiffness tensor are that the Lamé constants $\mu$ and $\lambda$ (or, with in our formulation, parameters $g$ and $k$ ) are strictly positive. In addition, they impose the constraint $\operatorname{tr}\left(\mathbf{B}^{2}\right)=3$. By virtue of the decomposition of eq. (18), the tensor B derives from a scalar parameter $f$ that indirectly controls the intensity of anisotropy, and a traceless tensor governing the directional character of anisotropy. The normalisation should only affect the scalar value, such that $0<f \leq 1$. The constraint $\operatorname{tr}\left(\mathbf{B}^{2}\right)=3$ corresponds to the requirement that $\mathbf{F}: \mathbf{F}$ $=3-3 f^{2}$.

Here we propose an alternative normalisation $\operatorname{tr}(\mathbf{B})=3$ that, by the decomposition of $\mathbf{B}$, leads simply to $f=1$.

The above constraints on $\mathbf{B}$ can be straightforwardly rewritten in terms of the anisotropy tensor $\mathbf{a}$. In fact, recalling the relation $\mathbf{B}=\mathbf{a}^{2}$, the rules $\operatorname{tr}\left(\mathbf{B}^{2}\right)=3$ and $\operatorname{tr}(\mathbf{B})=3$ become $\operatorname{tr}\left(\mathbf{a}^{4}\right)=3$ and $\operatorname{tr}\left(\mathbf{a}^{2}\right)=3$, respectively.

There is no reason to prefer a priori one or another of the above constraints, hence the user can choose the most convenient for a specific application. 


\subsection{Relationships with existing models}

\subsubsection{Graham \& Houlsby (1983)}

Graham and Houlsby [21] proposed a linear anisotropic elastic model formulated in triaxial space to describe transverse isotropy. They introduced anisotropy to the elastic stiffness tensor by multiplying the stiffness coefficients in the horizontal direction (supposing the horizontal plane as that of isotropy) by a scalar factor $\alpha$, whilst maintaining the symmetry of the stiffness matrix. Expressing the elastic stiffness tensor in terms of the bulk modulus $K$ and the shear modulus $G$, choosing the vertical direction as 1 their model is equivalent to:

$$
\left\{\begin{array}{l}
\sigma_{1} \\
\sigma_{2} \\
\sigma_{3}
\end{array}\right\}=\left[\begin{array}{ccc}
\alpha^{-\frac{4}{3}}\left(K+\frac{4}{3} G\right) & \alpha^{-\frac{1}{3}}\left(K-\frac{2}{3} G\right) & \alpha^{-\frac{1}{3}}\left(K-\frac{2}{3} G\right) \\
\alpha^{-\frac{1}{3}}\left(K-\frac{2}{3} G\right) & \alpha^{\frac{2}{3}}\left(K+\frac{4}{3} G\right) & \alpha^{\frac{2}{3}}\left(K-\frac{2}{3} G\right) \\
\alpha^{-\frac{1}{3}}\left(K-\frac{2}{3} G\right) & \alpha^{\frac{2}{3}}\left(K-\frac{2}{3} G\right) & \alpha^{\frac{2}{3}}\left(K+\frac{4}{3} G\right)
\end{array}\right]\left\{\begin{array}{l}
\varepsilon_{1} \\
\varepsilon_{2} \\
\varepsilon_{3}
\end{array}\right\}
$$

For $\alpha>1$ the material is stiffer horizontally than vertically, and the ratio of the stiffnesses in the horizontal and vertical directions is $\alpha^{2}$, namely the ratio of the second and the first diagonal terms of the matrix. The parameter $\alpha$ is a direct measure of the anisotropy of the material and for $\alpha=1$ isotropic elasticity is recovered.

In order to compare the above model to our one for the linear anisotropic case, we specialise eq. (23) for triaxial conditions. Furthermore, in the principal direction reference system, we assume the tensor $\mathbf{a}$ is diagonal and coaxial with the principal stresses and strains, and characterised by its eigenvalues $a_{1}, a_{2}$ and $a_{3}$. The result is:

$$
\left\{\begin{array}{l}
\sigma_{1} \\
\sigma_{2} \\
\sigma_{3}
\end{array}\right\}=p_{r}\left[\begin{array}{ccc}
\left(k+\frac{4}{3} g\right) a_{1}^{4} & \left(k-\frac{2}{3} g\right) a_{2}^{2} a_{1}^{2} & \left(k-\frac{2}{3} g\right) a_{3}^{2} a_{1}^{2} \\
\left(k-\frac{2}{3} g\right) a_{1}^{2} a_{2}^{2} & \left(k+\frac{4}{3} g\right) a_{2}^{4} & \left(k-\frac{2}{3} g\right) a_{3}^{2} a_{2}^{2} \\
\left(k-\frac{2}{3} g\right) a_{1}^{2} a_{3}^{2} & \left(k-\frac{2}{3} g\right) a_{2}^{2} a_{3}^{2} & \left(k+\frac{4}{3} g\right) a_{3}^{4}
\end{array}\right]\left\{\begin{array}{l}
\varepsilon_{1} \\
\varepsilon_{2} \\
\varepsilon_{3}
\end{array}\right\}
$$

Noting that $a_{1} \neq a_{2}=a_{3}$ because of the transverse isotropy, it is possible to write the anisotropy tensor a in a multiplicative form:

$$
\mathbf{a}=\left[\begin{array}{ccc}
a & 0 & 0 \\
0 & y a & 0 \\
0 & 0 & y a
\end{array}\right]
$$


where the parameter $y$ is the ratio of the stiffness along the two relevant principal directions. Depending on the choice of normalisation, this parameter takes different forms for the three different normalisations:

$$
\begin{aligned}
& \operatorname{det}(\mathbf{a})=1 \quad \rightarrow \quad y=a^{-\frac{3}{2}} \\
& \operatorname{tr}\left(\mathbf{a}^{2}\right)=3 \quad \rightarrow \quad y=\sqrt{\frac{3-a^{2}}{2 a^{2}}} \\
& \operatorname{tr}\left(\mathbf{a}^{4}\right)=3 \quad \rightarrow \quad y=\left(\frac{3-a^{4}}{2 a^{4}}\right)^{\frac{1}{4}}
\end{aligned}
$$

Specialising eq. (29) to the case of transverse anisotropy and comparing with eq. (28), it follows that $y=\alpha^{2}$, irrespective of the normalisation for $\mathbf{a}$, indicating that our proposed formulation encompasses that of Graham and Houlsby [21] as a special case.

\subsubsection{Lashkari (2010)}

Lashkari [33] proposed an extension of a bounding surface plasticity model [12] to account for the anisotropic elastic behaviour of sands. This feature was added by introducing a deviatoric, symmetric second order fabric tensor $\mathbf{F}$, which has the same character as described above. Following the expression for the elastic stiffness tensor developed by Cowin [10] and incorporating only the first order terms in F, Lashkari [33] obtained:

$$
\mathbf{D}=\left(K-\frac{2}{3} G\right)\left[\mathbf{I} \otimes \mathbf{I}+\omega_{1}(\mathbf{I} \otimes \mathbf{F}+\mathbf{F} \otimes \mathbf{I})\right]+2 G \mathbf{I} \underline{\otimes} \mathbf{I}+2 G \omega_{2}(\mathbf{I} \underline{\otimes} \mathbf{F}+\mathbf{F} \underline{\otimes} \mathbf{I})
$$

where $\omega_{1}$ and $\omega_{2}$ are scalar material constants and $K$ and $G$ are the elastic bulk and shear moduli. Since, in this model, the moduli are assumed to be non-linearly dependent on stress, the elastic formulation is hypoelastic.

It is possible to demonstrate that the free energy function leading to eq. (32) for its linear case (i.e. when the moduli are constants) would be:

$$
E(\boldsymbol{\varepsilon}, \mathbf{F})=\frac{1}{2}\left(K-\frac{2}{3} G\right)\left\{\operatorname{tr}^{2}(\boldsymbol{\varepsilon})+2 \omega_{1} \operatorname{tr}(\boldsymbol{\varepsilon}) \operatorname{tr}(\mathbf{F} \boldsymbol{\varepsilon})\right\}+G \operatorname{tr}\left(\boldsymbol{\varepsilon}^{2}\right)+2 G \omega_{2} \operatorname{tr}\left(\mathbf{F} \boldsymbol{\varepsilon}^{2}\right)
$$

Comparing the structure of eq. (33) to eq. (25) it is apparent that the latter represents a more general formulation, as that by Lashkari [33] neglects the two invariants $\operatorname{tr}^{2}(\mathbf{F} \boldsymbol{\varepsilon})$ and $\operatorname{tr}\left[(\mathbf{F} \boldsymbol{\varepsilon})^{2}\right]$. In fact, referring to the general free energy expression of eq. (13) proposed by Cowin [10], this corresponds to setting the constants $c_{3}$ and $c_{6}$ to zero. As a consequence, the expression for the stiffness tensor of eq. (32) does not include the terms in $\mathbf{F} \otimes \mathbf{F}$ and $\mathbf{F} \underline{\otimes} \underline{\mathbf{F}}$. 


\subsubsection{Mašín \& Rott (2014)}

Mašín and Rott [36] formulated a linear anisotropic elastic model using the representation theorems for transversely isotropic tensor functions. Defining the second order fabric tensor as $\mathbf{p}=\boldsymbol{n} \otimes \boldsymbol{n}$, where $\boldsymbol{n}$ is a unit normal vector to the plane of symmetry, their fourth order elastic stiffness takes the form:

$$
\mathbf{D}=b_{1} \mathbf{I} \otimes \underline{\mathbf{I}}+b_{2} \mathbf{I} \otimes \mathbf{I}+b_{3}(\mathbf{I} \otimes \mathbf{p}+\mathbf{p} \otimes \mathbf{I})+b_{4}(\mathbf{I} \otimes \bar{\otimes} \mathbf{p}+\mathbf{p} \bar{\otimes} \mathbf{I})+b_{5} \mathbf{p} \otimes \mathbf{p}
$$

where $b_{i}, i=1,5$ are material constants, possibly expressed in terms of the Young's and shear moduli and the Poisson's ratios in the plane of symmetry and along the orthogonal direction. The corresponding strain energy function is:

$$
E(\boldsymbol{\varepsilon}, \mathbf{p})=\frac{b_{1}}{2} \operatorname{tr}\left(\boldsymbol{\varepsilon}^{2}\right)+\frac{b_{2}}{2} \operatorname{tr}^{2}(\boldsymbol{\varepsilon})+b_{3} \operatorname{tr}(\boldsymbol{\varepsilon}) \operatorname{tr}(\mathbf{p} \boldsymbol{\varepsilon})+b_{4} \operatorname{tr}\left(\mathbf{p} \varepsilon^{2}\right)+\frac{b_{5}}{2} \operatorname{tr}^{2}(\mathbf{p} \boldsymbol{\varepsilon})
$$

To compare the proposed linear anisotropic formulation to that of Mašín and Rott [36], the fabric tensor a can be expressed by its spectral decomposition:

$$
\mathbf{a}=\sum_{i=1}^{3} a_{i} \boldsymbol{q}_{i} \otimes \boldsymbol{q}_{i}
$$

where $\boldsymbol{q}_{i}$ are the orthonormal eigenvectors defining the direction of orthotropy and $a_{i}$ are the eigenvalues of $\mathbf{a}$. Denoting the dyadic product of the eigenvectors as $\mathbf{M}_{i}=\boldsymbol{q}_{i} \otimes \boldsymbol{q}_{i}$, it follows that $\mathbf{M}_{1}+\mathbf{M}_{2}+\mathbf{M}_{3}=\mathbf{I}$. Specialising eq. (36) to the case of transverse isotropy, with $a_{1} \neq a_{2}=a_{3}$, one can rewrite eq. (22) retaining only one of the three eigenprojections $\mathbf{M}_{i}$, denoted by $\mathbf{M}$. Noting that when $\mathbf{M}$ is expressed by a dyadic product it follows that $\operatorname{tr}\left[(\mathbf{M} \boldsymbol{\varepsilon})^{2}\right]=\operatorname{tr}^{2}(\mathbf{M} \boldsymbol{\varepsilon})$ (e.g. Itskov [29]) and taking into account the related property $\mathbf{M}^{k}=\mathbf{M}$ for $k=1,2, \ldots n$, this leads to:

$$
E(\boldsymbol{\varepsilon}, \mathbf{M})=\frac{c_{1}}{2} \operatorname{tr}\left(\boldsymbol{\varepsilon}^{2}\right)+\frac{c_{2}}{2} \operatorname{tr}^{2}(\boldsymbol{\varepsilon})+c_{3} \operatorname{tr}(\boldsymbol{\varepsilon}) \operatorname{tr}(\mathbf{M} \boldsymbol{\varepsilon})+c_{4} \operatorname{tr}\left(\mathbf{M} \boldsymbol{\varepsilon}^{2}\right)+\frac{c_{5}}{2} \operatorname{tr}^{2}(\mathbf{M} \boldsymbol{\varepsilon})
$$

For the transverse isotropy, the strain energy involves five coefficients $c_{i}$, which can be expressed as function of the material constants $k$ and $g$ and the eigenvalues $a_{1}$ and $a_{2}$ as follows:

$$
\begin{aligned}
& c_{1}=2 p_{r} g a_{2}^{4} \quad ; \quad c_{2}=p_{r}\left(k-\frac{2}{3} g\right) a_{2}^{4} ; c_{3}=p_{r}\left(k-\frac{2}{3} g\right) a_{2}^{2}\left(a_{1}^{2}-a_{2}^{2}\right) \\
& c_{4}=2 p_{r} g a_{2}^{2}\left(a_{1}^{2}-a_{2}^{2}\right) ; \quad c_{5}=p_{r}\left(k+\frac{4}{3} g\right)\left(a_{1}^{2}-a_{2}^{2}\right)^{2}
\end{aligned}
$$

Comparing eq. (37) and (35) it follows that the formulations are equivalent since both $\mathbf{M}$ and $\mathbf{p}$ are defined as a dyadic product of a unit normal vector, and the strain energy functions are expressed in terms of the same invariants of the fabric and strain tensors. Nonetheless, it is worth recalling that 
the Mašín and Rott [36] formulation is only valid for linear transverse isotropy, therefore it represents a particular case of the linear version of our proposed formulation. For $\mathbf{M}=\mathbf{0}$ (or equivalently $\mathbf{p}=\mathbf{0}$ ) the linear isotropic formulation is recovered.

\subsubsection{Zhao \& Gao (2015)}

Zhao and Gao [47] proposed, within the framework of hypoelasticity, an anisotropic elastic stiffness tensor expressed in terms of a deviatoric, symmetric second order fabric tensor $\mathbf{F}$. This tensor is employed to describe the fabric anisotropy in sand and characterises the isotropic, transverse isotropic and orthotropic reversible response of the model. They derived the following simplified expression for the stiffness tensor, starting from the work of Cowin [10], neglecting the second and higher order terms of $\mathbf{F}$, and obtaining an expression similar to that proposed Lashkari [33]:

$$
\mathbf{D}=\left(K-\frac{2}{3} G\right)\left[\mathbf{I} \otimes \mathbf{I}+\frac{1}{2}(\mathbf{I} \otimes \mathbf{F}+\mathbf{F} \otimes \mathbf{I})\right]+2 G \mathbf{I} \otimes \mathbf{I}+G(\mathbf{I} \otimes \underline{\mathbf{F}}+\mathbf{F} \underline{\otimes} \mathbf{I})
$$

where $K$ and $G$ denote the elastic bulk modulus and the elastic shear modulus.

In a similar way as above, the free energy potential leading to the elastic stiffness tensor in eq. (39) is easily defined as:

$$
E(\boldsymbol{\varepsilon}, \mathbf{F})=\frac{1}{2}\left(K-\frac{2}{3} G\right)\left\{\operatorname{tr}^{2}(\boldsymbol{\varepsilon})+\operatorname{tr}(\boldsymbol{\varepsilon}) \operatorname{tr}(\mathbf{F} \boldsymbol{\varepsilon})\right\}+G \operatorname{tr}\left(\boldsymbol{\varepsilon}^{2}\right)+G \operatorname{tr}\left(\mathbf{F} \boldsymbol{\varepsilon}^{2}\right)
$$

Once again, comparing eq. (25) and (40) it emerges that the Zhao and Gao [47] expression omits some of the terms of our formulation. 


\section{The complete non-linear anisotropic hyperelastic formulation}

In this section, the complete non-linear anisotropic hyperelastic model is discussed. The anisotropic behaviour of soils, stemming from the microstructural characteristics of the material, is introduced in the model by the constant, symmetric second order fabric tensor a, subjected to the same restrictions described in the previous sections. In detail, employing the two mixed invariants $\operatorname{tr}(\mathbf{a \varepsilon a})$ and $\operatorname{tr}\left[\left(\mathbf{a} \varepsilon^{s y m} \mathbf{a}\right)^{2}\right]$, one can specialise the free energy potential of eq. (6) as follows:

$$
\begin{aligned}
E(\boldsymbol{\varepsilon}, \mathbf{a}) & =\frac{p_{r}}{k(2-n)} k^{\frac{2-n}{2-2 n}}(1-n)^{\frac{2-n}{2-2 n}}\left\{\left[k(1-n)-\frac{2}{3} g\right] \operatorname{tr}^{2}(\mathbf{a} \mathbf{\varepsilon a})+2 g \operatorname{tr}\left[\left(\mathbf{a} \varepsilon^{s y m} \mathbf{a}\right)^{2}\right]\right\}^{\frac{2-n}{2-2 n}}= \\
& =\frac{p_{r}}{k(2-n)} \bar{r}_{0}^{\frac{2-n}{1-n}}
\end{aligned}
$$

The stress tensor is thus obtained differentiating the free energy function with respect to the strain:

$$
\boldsymbol{\sigma}=p_{r} \bar{r}_{0}^{\frac{n}{1-n}}\left\{\left[k(1-n)-\frac{2}{3} g\right] \operatorname{tr}(\mathbf{a} \mathbf{\varepsilon}) \mathbf{a}^{2}+2 g \mathbf{a}^{2} \boldsymbol{\varepsilon}^{s y m} \mathbf{a}^{2}\right\}
$$

and, with further differentiation, the stiffness tensor is:

$$
\begin{aligned}
\mathbf{D} & =p_{r}\left[k(1-n)-\frac{2}{3} g\right]\left\{k \bar{r}_{0}^{\frac{3 n-2}{1-n}} n\left[k(1-n)-\frac{2}{3} g\right] \operatorname{tr}^{2}(\mathbf{a} \boldsymbol{\varepsilon})+\bar{r}_{0}^{\frac{n}{1-n}}\right\} \mathbf{a}^{2} \otimes \mathbf{a}^{2}+ \\
& +4 p_{r} k \bar{r}_{0}^{\frac{3 n-2}{1-n}} n g^{2}\left(\mathbf{a}^{2} \boldsymbol{\varepsilon}^{s y m} \mathbf{a}^{2} \otimes \mathbf{a}^{2} \boldsymbol{\varepsilon}^{\text {sym }} \mathbf{a}^{2}\right)+ \\
& +2 p_{r} k \bar{r}_{0}^{\frac{3 n-2}{1-n}} n g\left[k(1-n)-\frac{2}{3} g\right] \operatorname{tr}(\mathbf{a} \boldsymbol{\varepsilon a})\left(\mathbf{a}^{2} \boldsymbol{\varepsilon}^{\text {sym }} \mathbf{a}^{2} \otimes \mathbf{a}^{2}+\mathbf{a}^{2} \otimes \mathbf{a}^{2} \boldsymbol{\varepsilon}^{s y m} \mathbf{a}^{2}\right)+ \\
& +2 p_{r} \bar{r}_{0}^{\frac{n}{1-n}} g\left(\mathbf{a}^{2} \otimes \mathbf{a}^{2}\right)
\end{aligned}
$$

Note that this latter equation only applies for the case where a is a constant, such that only a fixed inherent anisotropy (i.e. not an anisotropy which evolves with strain) is accounted for. In a more general case, as for example in elasto-plastic materials, anisotropy can evolve as a function of tensorial internal variables (e.g. plastic strains), leading to a form of anisotropic elasto-plastic coupling which is not investigated in this work. Nonetheless, it is worth mentioning that in such a circumstance, following Maier and Hueckel [35] and Collins and Houlsby [9], eq. (43) would describe the instantaneous reversible stiffness of the soil.

The proposed formulation accounts for both inherent and stress-induced anisotropy. In fact, it not only reproduces the non-linear stress-dependent stiffness and the related evolving directional elastic properties with the current stress/strain state, but also allows modelling of the permanent anisotropic 
characteristics via the a tensor. All the above features are enriched by the energy-based derivation of the formulation, which ensures its thermodynamic consistency.

The dual stress-based formulation is reported in the Appendix in terms of complementary free energy and strain and compliance tensors.

Eqs. (41) - (43) can also be expressed in terms of the $\mathbf{B}$ tensor, recalling that $\mathbf{B}=\mathbf{a}^{2}$. Furthermore, as in Section 4 for the linear case, the above non-linear anisotropic formulation can be rewritten adopting for the decomposition of the fabric tensor $\mathbf{B}$ as in eq. (18). For the sake of conciseness, the resulting equations are reported in the Appendix.

\subsection{Comparison with existing formulations}

\subsubsection{Cudny \& Partyka (2017)}

Cudny and Partyka [11] proposed an extension of the non-linear isotropic hyperelastic model developed by Vermeer [43] to describe the transverse isotropic behaviour of soils. Following an approach similar to that of Mašín and Rott [36], they introduced into the original isotropic formulation a second order fabric tensor $\mathbf{N}=\boldsymbol{v} \otimes \boldsymbol{v}$, defined as the dyadic product of a unit vector $\boldsymbol{v}$. In detail, they modify Vermeer's complementary energy function by introducing the mixed invariant $\operatorname{tr}\left(\mathbf{N} \boldsymbol{\sigma}^{2}\right)$ as follows:

$$
C(\boldsymbol{\sigma}, \mathbf{N})=\frac{3 p_{r e f}^{1-\beta}}{2 G_{0}^{r e f}(1+\beta)}\left[\frac{c_{1}}{3} \operatorname{tr}\left(\boldsymbol{\sigma}^{2}\right)+\frac{c_{2}}{3} \operatorname{tr}\left(\mathbf{N} \boldsymbol{\sigma}^{2}\right)\right]^{\frac{1+\beta}{2}}
$$

where $G_{0}^{r e f}$ is the reference shear modulus at the reference mean pressure $p_{\text {ref }}$ and $\beta$ is the material constant controlling the non-linear dependence of the elastic stiffness with the state of stress. If $c_{1}=$ 1 and $c_{2}=0$ or $c_{1}=1$ and $\mathbf{N}=\mathbf{0}$ the structural anisotropy is deactivated and the Vermeer isotropic formulation is recovered.

In order to compare the Cudny and Partyka [11] model to our one, we rewrite the complementary energy reported in the Appendix (eq. (A1)) adopting spectral decomposition, analogously to the approach in section 4.2.3. In particular, denoting the eigenprojections of $\mathbf{a}^{-1}$ as $\mathbf{N}_{i}$ the fabric tensor can be written such that

$$
\mathbf{a}^{-1}=\sum_{i=1}^{3} \frac{1}{a_{i}}\left(\boldsymbol{q}_{i} \otimes \boldsymbol{q}_{i}\right)^{-1}=\sum_{i=1}^{3} \frac{1}{a_{i}} \mathbf{N}_{i}
$$


Under the hypothesis of transverse isotropy $a_{1} \neq a_{2}=a_{3}$ and $\mathbf{N}_{1} \neq \mathbf{N}_{2}=\mathbf{N}_{3}$. In addition, recalling that $\mathbf{N}_{1}+\mathbf{N}_{2}+\mathbf{N}_{3}=\mathbf{I}$, only one of the three $\mathbf{N}_{i}$ is retained, simply indicated with $\mathbf{N}$. Specialising eq. (36) for this case the energy reads:

$$
\begin{aligned}
C(\boldsymbol{\sigma}, \mathbf{N}) & =\frac{1}{p_{r}{ }^{1-n} k(1-n)(2-n)}\left[c_{1} \operatorname{tr}^{2}(\boldsymbol{\sigma})+c_{2} \operatorname{tr}\left(\boldsymbol{\sigma}^{2}\right)+c_{3} \operatorname{tr}(\boldsymbol{\sigma}) \operatorname{tr}(\mathbf{N} \boldsymbol{\sigma})+\right. \\
& \left.+c_{4} \operatorname{tr}^{2}(\mathbf{N} \boldsymbol{\sigma})+c_{5} \operatorname{tr}\left(\mathbf{N} \boldsymbol{\sigma}^{2}\right)\right]^{\frac{2-n}{2}}
\end{aligned}
$$

with the five coefficients $c_{i}$ functions of material constants $k$ and $g$ and the eigenvalues $a_{1}^{-1}$ and $a_{2}^{-1}$ of the tensor $\mathbf{a}^{-1}$.

$$
\begin{aligned}
& c_{1}=\left(\frac{1}{9}-\frac{k(1-n)}{6 g}\right) a_{2}^{-4} ; \quad c_{2}=\frac{k(1-n)}{2 g} a_{2}^{-4} ; \quad c_{3}=2\left(\frac{1}{9}-\frac{k(1-n)}{6 g}\right) a_{2}^{-2}\left(a_{1}^{-2}-a_{2}^{-2}\right) \\
& c_{4}=\left(\frac{1}{9}+\frac{k(1-n)}{3 g}\right)\left(a_{1}^{-2}-a_{2}^{-2}\right)^{2} ; c_{5}=\frac{k(1-n)}{g} a_{2}^{-2}\left(a_{1}^{-2}-a_{2}^{-2}\right)
\end{aligned}
$$

Comparing the complementary energy functions of eqs. (44) and (46) it is apparent that the way in which the non-linearisation is introduced is similar, though the model by Cudny and Partyka [11] does not include some of the terms in the model proposed here. In fact, in the latter the three additional invariants $\operatorname{tr}^{2}(\boldsymbol{\sigma}), \operatorname{tr}(\boldsymbol{\sigma}) \operatorname{tr}(\mathbf{N} \boldsymbol{\sigma})$ and $\operatorname{tr}^{2}(\mathbf{N} \boldsymbol{\sigma})$ appear. It is also worth recalling that the Cudny and Partyka [11] model only holds for the transverse isotropy case.

\subsubsection{Gajo \& Bigoni (2008)}

Gajo and Bigoni [17] proposed a non-linear anisotropic hyperelastic model, developed within the framework of elasto-plasticity. As discussed in this work, the model can describe the non-linear stress dependency of the elastic stiffness and, at the same time, includes a tensor-based description of the structural anisotropy (by the tensor B), which in their formulation evolves with the plastic strains. Limiting attention to the pure reversible behaviour (i.e., in absence of plastic deformation), the fabric tensor is constant and their free energy potential assumes the form:

$$
E(\boldsymbol{\varepsilon})=\alpha[\operatorname{tr}(\mathbf{B} \boldsymbol{\varepsilon})]^{n}+\beta\left[\operatorname{tr}\left((\mathbf{B} \boldsymbol{\varepsilon})^{2}\right)\right]^{l}
$$

where $\alpha, \beta, n$ and $l$ are model parameters. For $n=2$ and $l=1$ the linear anisotropy described by Bigoni and Loret [3] is recovered and when $\mathbf{B}=\mathbf{I}$ the elastic behaviour becomes isotropic.

Following the hyperelastic framework, differentiating with respect to the strains, Gajo and Bigoni [17] obtain the stress tensor: 


$$
\boldsymbol{\sigma}=\alpha n[\operatorname{tr}(\mathbf{B} \boldsymbol{\varepsilon})]^{n-1} \mathbf{B}+2 l \beta\left[\operatorname{tr}\left((\mathbf{B} \boldsymbol{\varepsilon})^{2}\right)\right]^{l-1} \mathbf{B} \boldsymbol{\varepsilon} \mathbf{B}
$$

and, by further differentiation, the elastic stiffness tensor:

$$
\begin{aligned}
\mathbf{D} & =\alpha n(n-1)[\operatorname{tr}(\mathbf{B} \boldsymbol{\varepsilon})]^{n-2} \mathbf{B} \otimes \mathbf{B}+4 l \beta(l-1)\left[\operatorname{tr}\left((\mathbf{B} \boldsymbol{\varepsilon})^{2}\right)\right]^{l-2} \mathbf{B} \boldsymbol{\varepsilon} \mathbf{B} \otimes \mathbf{B} \boldsymbol{\varepsilon} \mathbf{B}+ \\
& +2 \beta l\left[\operatorname{tr}\left((\mathbf{B} \boldsymbol{\varepsilon})^{2}\right)\right]^{l-1}(\mathbf{B} \underline{\otimes} \mathbf{B})
\end{aligned}
$$

The main difference between the free energy potential of eq. (48) and that proposed in this paper (eq. (41)) is in the description of non-linearity: Gajo and Bigoni [17] adopt two different exponents, $n$ and $l$, acting separately on the two mixed invariants, while in our case quadratic expression for a linear material is raised to a power with a single exponent.

Comparing the two formulations is not straightforward in the general anisotropic case. Nonetheless, as the main difference is in the treatment of non-linearity, in the following the comparison is illustrated for the simplified isotropic case $(\mathbf{B}=\mathbf{I})$ under triaxial conditions. In this case eq. (48) can be rewritten as:

$$
E\left(\varepsilon_{v}, \varepsilon_{s}\right)=\alpha \varepsilon_{v}^{n}+\beta\left(\frac{3}{2} \varepsilon_{s}^{2}+\frac{1}{3} \varepsilon_{v}^{2}\right)^{l}
$$

so that the terms of the stiffness matrix, following the structure of eq. (9), are:

$$
\begin{aligned}
& D_{11}=\beta l(l-1)\left(\frac{3}{2} \varepsilon_{s}^{2}+\frac{1}{3} \varepsilon_{v}^{2}\right)^{l-2}\left[\frac{4}{9} \varepsilon_{v}^{2}+\frac{2}{3} \frac{1}{l-1}\left(\frac{3}{2} \varepsilon_{s}^{2}+\frac{1}{3} \varepsilon_{v}^{2}\right)\right]+\alpha n(n-1) \varepsilon_{v}^{n-2} \\
& D_{12}=D_{21}=\beta l(l-1)\left(\frac{3}{2} \varepsilon_{s}^{2}+\frac{1}{3} \varepsilon_{v}^{2}\right)^{l-2} 2 \varepsilon_{s} \varepsilon_{v} \\
& D_{22}=\beta l(l-1)\left(\frac{3}{2} \varepsilon_{s}^{2}+\frac{1}{3} \varepsilon_{v}^{2}\right)^{l-2}\left[9 \varepsilon_{s}^{2}+\frac{3}{l-1}\left(\frac{3}{2} \varepsilon_{s}^{2}+\frac{1}{3} \varepsilon_{v}^{2}\right)\right]
\end{aligned}
$$

Comparing the isotropic elastic stiffness obtained using the proposed model (eq. (10)) and the Gajo and Bigoni [17] case, it can be noted that the major difference lies in the term $D_{11}$. Evaluating the ratio between the two diagonal terms of the stiffness matrix for an isotropic state of strain/stress $\left(\varepsilon_{s}=\right.$ 0), gives for Gajo and Bigoni [17]:

$$
\frac{D_{11}}{D_{22}}=\frac{\alpha n(n-1) \varepsilon_{v}^{n-2}}{3 \beta l\left(\frac{1}{3} \varepsilon_{v}^{2}\right)^{l-1}}+(l-1) \frac{4}{27}\left(\frac{1}{3}\right)^{l-2}+\frac{2}{9}
$$

and for our proposed model:

$$
\frac{D_{11}}{D_{22}}=\frac{k}{3 g}
$$


This ratio can be interpreted as the ratio between the bulk modulus $K$ and the shear modulus $G$, which is a function of the Poisson's ratio. The difference between the formulations is substantial: in our proposed model the above ratio is constant, while in the model by Gajo and Bigoni [17] it depends on the volumetric strain (except for the special case of $l=n / 2$ ). This implies a constant Poisson's ratio for the proposed formulation under isotropic compression, while in that of Gajo and Bigoni [17] the Poisson's ratio may either be constant (with $l=n / 2$ ) or evolve during this stress path.

In recent years the improvement of laboratory testing devices has allowed investigation of the small strain behaviour of soils with relatively high accuracy. In particular, triaxial or hollow cylinder apparatus fitted with high resolution local transducers and bender elements has provided a wide and consistent set of experimental data on the reversible response of different materials in the small strain range (i.e. in the reversible regime). Many attempts have been made to evaluate Poisson's ratio under different loading conditions. The most reliable results are those based on the direct measurements of vertical and horizontal strains, complemented by shear wave velocity measurements. For example, different sands have been tested by these techniques, under both static and dynamic conditions $[7,13$, $15,24,28,32]$. Despite the expected variation exhibited by the experimental data, all the above results indicate that along sufficiently extended isotropic stress paths each Poisson's ratio tends towards an approximately constant value, as for example illustrated in Fig. 2 for the Hostun sand [15]. This result is consistent with the prediction by the elastic formulation discussed in this paper.

Another drawback of the model formulated by Gajo and Bigoni [17] is the absence of a defined complementary energy function: Gajo and Bigoni [17] propose an approximate expression, whose consistency with their free energy function is only verified for a specific set of material parameters. Conversely, in our proposed model the complementary energy function is always valid as it stems from a Legendre transform. The definition of a complementary energy function is important as it allows the constitutive equations to be defined in both stiffness and compliance forms. This aspect is useful in the context of the implicit numerical integration of elasto-plastic constitutive models, as discussed for example by Borja et al. [6]; Tamagnini et al. [41]; Amorosi et al. [1].

\section{Calibration and model performance}

In this section, the strategy for parameter calibration, and the predictive capability of the model are discussed for both clays and sands.

The isotropic model parameters consist of three scalar quantities $g, k$, and $n$ controlling the magnitude of the components of the elastic stiffness tensor and their dependence on the current state 
of stress. These three constants can be calibrated from the evolution of the shear elastic modulus $G$ and the bulk modulus $K$ (or equivalently Young's modulus $E$ ) with the stress level for an isotropic material, or along a specific stress path. The inherent (structural) anisotropic behaviour is controlled by the tensor a, which in the most general case requires five further parameters to be calibrated (as the tensor is (a) symmetric and (b) normalised). Nevertheless, it is often the case that the principal directions of anisotropy are known a priori. For instance, under the additional restrictive hypothesis of transverse isotropy with a vertical axis of symmetry, only one anisotropy parameter has to be defined.

The elastic anisotropic behaviour of soils can be investigated experimentally by in situ and/or laboratory tests. The measurement of the shear wave velocities propagated along different directions and polarised in three orthogonal planes allows determination of the corresponding small strain shear moduli. This can be achieved through dynamic field techniques such as cross-hole tests [8, 23] or measurements with laboratory bender elements. Combining these latter with small strain triaxial static and dynamic tests, and assuming the soil to be an elastic transverse isotropic material, it is possible to detect all the terms of the elastic stiffness tensor. More sophisticated laboratory devices, such as the hollow cylinder apparatus, allow direct estimates the five independent parameters of a crossanisotropic material.

\section{Sands}

Kuwano and Jardine [32] investigated the elastic anisotropic behaviour of the Ham River sand, a uniform, medium-sized and sub-angular-shaped quartz sand. They used large specimens, 100mm in diameter and $200 \mathrm{~mm}$ height, obtained by air pluviation and then water saturated. They performed triaxial tests with high-resolution axial and radial LVDT transducers and bender elements. Fig. 3 illustrates the anisotropic compression path imposed on each specimen, consisting of an initial increase of the stress ratio, from the isotropic stress state of $30 \mathrm{kPa}$ up to that corresponding to $K_{0}=$ 0.45 , followed by a anisotropic consolidation at constant stress ratio.

Under the hypothesis of cross anisotropy, they illustrate the evolution of the terms of the instantaneous elastic stiffness matrix with the mean effective pressure. In detail, they obtained the Young's moduli $E_{v}$ and $E_{h}$ in the vertical and horizontal directions through static tests and, for the same states, the shear moduli $G_{h h}, G_{h v}$ and $G_{v h}$ from bender element probing. In Fig. 4 the experimental data are shown with dots together with the simulations of our model. The material exhibits a non-negligible degree of structural anisotropy, as indicated by the different Young's and shear moduli observed along different directions at the very beginning of the probing, carried out 
once the required anisotropic stress state had been first achieved. The results also demonstrate a welldefined non-linear dependency of the stiffness on the current stress state.

The parameters of the proposed hyperelastic model have been calibrated with reference to the above experimental data under the hypothesis of cross anisotropy. Reference vertical axis of symmetry (coincident with the axis of the triaxial samples) is assumed; the tensor a is diagonal with $a_{11} \neq a_{22}=a_{33}$, with principal direction 1 vertical. The ratio $\left(a_{22} / a_{11}\right)^{2}$ is equal to the shear moduli ratio $G_{h h} / G_{v h}$. The fitted parameters are reported in Table 5 .

Bellotti et al. [2] performed laboratory dynamic tests on the Italian Ticino River sand to investigate the anisotropic nature of the small strain stiffness for different effective stresses and void ratios. The dry cylindrical specimens of the uniform, coarse to medium-sized Ticino sand were reconstituted by pluviation and then subjected to radial triaxial stress paths characterised by different $K_{0}$ values. During the loading process Bellotti et al. [2] measured the shear wave velocities propagating along the vertical and horizontal directions and polarised in the vertical and horizontal planes; they also carried out measurements of the compression wave velocities along the same directions. As a consequence, in addition to the shear moduli $G_{h h}, G_{h v}$ and $G_{v h}$ they obtained, under the hypothesis of transverse isotropy, the Young's moduli $E_{v}$ and $E_{h}$. Because the samples were dry, the velocity of the compression waves provides data on the normal elastic stiffness components. Furthermore, they found that the velocity of waves propagated and polarised in the horizontal plane were independent of the direction of propagation, thus indicating isotropy of behaviour in the horizontal plane (i.e. cross anisotropy holds for those experiments).

Fig. 5-7 show the experimental results in terms of shear and Young's moduli against the mean effective pressure for a medium dense sand $(\mathrm{Dr}=41 \%)$ compressed along three different radial paths, characterised by $K_{0}$ of $0.5,1$ and 1.5 respectively. The figures also show the simulations of the model carried out for the parameters reported in Table 5 and ratios $a_{22} / a_{11}$ equal to $0.98,1.086$ and 1.118 for the corresponding increasing values of $K_{0}$.

The results obtained under isotropic stress conditions (Fig. 6) reveal the presence of an inherent anisotropy reflecting the internal structure of the material, possibly stemming from the grain shape and sample preparation technique. It is worth noting that different $K_{0}$ anisotropic compression paths induce different values for stress-induced anisotropy, leading for example to $E_{v}>E_{h}$ for $K_{0}<1$ and $E_{v}<E_{h}$ for $K_{0}>1$. The model simulations reproduce the observed response for a unique set of parameters $n, k$ and $g$ and an $a d$ hoc selection of the ratio $a_{22} / a_{11}$ for each investigated $K_{0}$ value to account for the corresponding structural modification of the internal microstructure. 
Clays

The elastic anisotropic behaviour of intact London clay has been experimentally investigated by Gasparre [18] and Gasparre et al. [19], who performed triaxial and hollow cylinder tests with highresolution axial and radial LVDT transducers and bender elements, under static and dynamic test conditions. They detected the terms of the instantaneous elastic stiffness matrix assuming the hypothesis of cross anisotropy. In a similar way to Kuwano and Jardine [32], they obtained the Young's moduli $E_{v}$ and $E_{h}$ along the vertical and horizontal directions through static and hybrid dynamic triaxial tests and the shear moduli $G_{h h}$ and $G_{h v}$ by bender element probing. In addition, a hollow cylinder apparatus (HCA) was used to perform measurements of the shear stiffness component $G_{v h}$ and the Young's moduli. They conclude that the results obtained using the two different experimental setups generally exhibit good agreement in terms of stiffness parameters.

The samples of the London clay, retrieved at different depths, were reconsolidated at the three different in situ stress states illustrated in Fig. 8 by black dots, based on the geological history of London clay as reported by Hight et al. [22].

London Clay is a heavily overconsolidated soil, characterised in situ by high horizontal effective stresses $\left(K_{0}>1\right)$ and a stiffer response in the plane of deposition, horizontal, than in the vertical direction. This is not only due to the anisotropic in situ stress state but also to the typical planar shape of the grains and the depositional processes, which lead to a preferred particle orientation along the horizontal plane.

In the laboratory, the consolidation process consisted of an initial isotropic stress path, followed by a constant mean effective pressure increment until the presumed in situ stress state was reached, as depicted in Fig. 9. The specimens were then subjected to the static and dynamic probing to detect their stiffness characteristics.

The model parameters are reported in Table 5 for an experimentally observed constant shear stiffness ratio $G_{h h} / G_{v h}=1.9$, which corresponds to the anisotropy ratio $a_{22} / a_{11}=1.378$.

The results are illustrated in Fig. 10, in terms of the evolution of Young's and shear moduli with the mean effective pressure. While the experimental data only refer to the three stress states of Fig. 9, the model simulations are extended to higher $p$ values following the shifted curved reported in Fig. 8. The scatter in the experimental results is probably due to the slightly different initial void ratios of the samples. It is worth recalling that the proposed model does not take into account the dependence of the stiffness on voids ratio or preconsolidation pressure, thus a single back-prediction is available for each stress state. The overall performance of the model reproduces the laboratory results in a satisfactory way. 
In addition, Gasparre [18] carried out tests on the same intact material compressed along the isotropic axis, determining the shear moduli at different mean effective pressures by bender elements polarised along perpendicular planes. In this case a larger number of experimental data are available and illustrated in Fig. 11, together with the corresponding model fitting. These show that the model is capable of capturing the observed trend in the evolution of the two components of the stiffness investigated in the experiments.

\section{Conclusions and future developments}

This paper explores the characteristics of the new non-linear anisotropic hyperelastic model presented in the HAR18 [27], with special emphasis on the similarities and differences of its formulation as compared to existing models. The model structure proves to encompass most but not all of the anisotropic elastic models for soils proposed in the literature, in some cases incorporating additional terms in the stiffness matrix. It is characterised by a modular structure, such that can be simplified to a linear isotropic one, depending on the modelling requirements and availability of data for the calibration of its parameters. The model employs only a small number of parameters which can readily be calibrated from standard tests. The predictive capability of the model is verified against accurate experimental results from the literature on both sands and a clay soil, making it flexible enough to be used in different soil mechanics problems.

Further research activity is required to account for elastic-plastic coupling effects, including the dependency of the stiffness on preconsolidation pressure, and (plastic) changes of void ratio, as well as the evolution of the anisotropy tensor during plastic deformation. 


\section{Appendix}

The complementary energy expressed via the tensor a is:

$$
\begin{aligned}
& C(\boldsymbol{\sigma}, \mathbf{a})=\frac{1}{p_{r}{ }^{1-n} k(1-n)(2-n)} \bar{p}_{0}^{2-n}= \\
& =\frac{1}{p_{r}{ }^{1-n} k(1-n)(2-n)}\left[\left(\frac{1}{9}-\frac{k(1-n)}{6 g}\right) \operatorname{tr}^{2}\left(\mathbf{a}^{-1} \boldsymbol{\sigma} \mathbf{a}^{-1}\right)+\frac{k(1-n)}{2 g} \operatorname{tr}\left[\left(\mathbf{a}^{-1} \boldsymbol{\sigma}^{s y m} \mathbf{a}^{-1}\right)^{2}\right]\right]^{\frac{2-n}{2}}
\end{aligned}
$$

The strain tensor is obtained differentiating the complementary energy with respect to the stress:

$$
\boldsymbol{\varepsilon}=\frac{1}{2 p_{r}^{1-n} k(1-n)} \bar{p}_{0}^{-n}\left[2\left(\frac{1}{9}-\frac{k(1-n)}{6 g}\right) \operatorname{tr}\left(\mathbf{a}^{-1} \boldsymbol{\sigma} \mathbf{a}^{-1}\right) \mathbf{a}^{-2}+\frac{k(1-n)}{g} \mathbf{a}^{-2} \boldsymbol{\sigma}^{\text {sym }} \mathbf{a}^{-2}\right]
$$

and, with further differentiation, the compliance tensor is:

$$
\begin{aligned}
\mathbf{C} & =\frac{1}{p_{r}{ }^{1-n} k(1-n)}\left\{\left(-\frac{n}{2}\right) \bar{p}_{0}^{-(n+2)} 2\left(\frac{1}{9}-\frac{k(1-n)}{6 g}\right) \operatorname{tr}^{2}\left(\mathbf{a}^{-1} \boldsymbol{\sigma} \mathbf{a}^{-1}\right)+\right. \\
& \left.+\bar{p}_{0}^{-n}\left(\frac{1}{9}-\frac{k(1-n)}{6 g}\right)\right\} \mathbf{a}^{-2} \otimes \mathbf{a}^{-2}+ \\
& -\frac{n}{2 p_{r}{ }^{1-n} g} \bar{p}_{0}^{-(n+2)}\left(\frac{1}{9}-\frac{k(1-n)}{6 g}\right) \operatorname{tr}\left(\mathbf{a}^{-1} \boldsymbol{\sigma} \mathbf{a}^{-1}\right)\left(\mathbf{a}^{-2} \otimes \mathbf{a}^{-2} \boldsymbol{\sigma}^{s y m} \mathbf{a}^{-2}+\mathbf{a}^{-2} \boldsymbol{\sigma}^{s y m} \mathbf{a}^{-2} \otimes \mathbf{a}^{-2}\right)+ \\
& -\frac{n}{4 p_{r}{ }^{1-n}} \bar{p}_{0}^{-(n+2)} \frac{k(1-n)}{g^{2}}\left(\mathbf{a}^{-2} \boldsymbol{\sigma}^{s y m} \mathbf{a}^{-2} \otimes \mathbf{a}^{-2} \boldsymbol{\sigma}^{s y m} \mathbf{a}^{-2}\right)+\frac{1}{2 p_{r}{ }^{1-n} g} \bar{p}_{0}^{-n} \mathbf{a}^{-2} \bar{\otimes} \mathbf{a}^{-2}
\end{aligned}
$$

Recalling that $\mathbf{B}=\mathbf{a}^{2}$, employing the two mixed invariants $\operatorname{tr}(\mathbf{B} \boldsymbol{\varepsilon})$ and $\operatorname{tr}\left[(\mathbf{B} \boldsymbol{\varepsilon})^{2}\right]$, one can equivalently rewrite the strain energy of eq. (41) as follows:

$$
\begin{aligned}
E(\boldsymbol{\varepsilon}, \mathbf{B}) & =\frac{p_{r}}{k(2-n)} \bar{r}^{\frac{2-n}{1-n}}= \\
& =\frac{p_{r}}{k(2-n)} k^{\frac{2-n}{2-2 n}}(1-n)^{\frac{2-n}{2-2 n}}\left\{\left[k(1-n)-\frac{2}{3} g\right] \operatorname{tr}^{2}(\mathbf{B} \boldsymbol{\varepsilon})+2 g \operatorname{tr}\left[\left(\mathbf{B} \boldsymbol{\varepsilon}^{\text {sym }}\right)^{2}\right]\right\}^{\frac{2-n}{2-2 n}}
\end{aligned}
$$

The stress tensor is thus obtained by differentiating the strain energy function with respect to the strain:

$$
\boldsymbol{\sigma}=p_{r} \bar{r}_{0}^{\frac{n}{1-n}}\left\{\left[k(1-n)-\frac{2}{3} g\right] \operatorname{tr}(\mathbf{B} \boldsymbol{\varepsilon}) \mathbf{B}+2 g \mathbf{B} \boldsymbol{\varepsilon}^{\text {sym }} \mathbf{B}\right\}
$$

and, with further differentiation, the stiffness tensor is: 


$$
\begin{aligned}
\mathbf{D} & =p_{r}\left[k(1-n)-\frac{2}{3} g\right]\left\{k \bar{r}_{0}^{\frac{3 n-2}{1-n}} n\left[k(1-n)-\frac{2}{3} g\right] \operatorname{tr}^{2}(\mathbf{B} \boldsymbol{\varepsilon})+\bar{r}_{0}^{\frac{n}{1-n}}\right\} \mathbf{B} \otimes \mathbf{B}+ \\
& +4 p_{r} k \bar{r}_{0}^{\frac{3 n-2}{1-n}} n g^{2}\left(\mathbf{B} \boldsymbol{\varepsilon}^{\text {sym }} \mathbf{B} \otimes \mathbf{B} \boldsymbol{\varepsilon}^{\text {sym }} \mathbf{B}\right)+ \\
& +2 p_{r} k \bar{r}_{0}^{\frac{3 n-2}{1-n}} n g\left[k(1-n)-\frac{2}{3} g\right] \operatorname{tr}(\mathbf{B} \boldsymbol{\varepsilon})\left(\mathbf{B} \boldsymbol{\varepsilon}^{\text {sym }} \mathbf{B} \otimes \mathbf{B}+\mathbf{B} \otimes \mathbf{B} \boldsymbol{\varepsilon}^{s y m} \mathbf{B}\right)+2 p_{r} \bar{r}_{0}^{\frac{n}{1-n}} g(\mathbf{B} \otimes \mathbf{\otimes})
\end{aligned}
$$

Analogously, the complementary energy expressed via the tensor $\mathbf{B}$ is:

$$
\begin{aligned}
& C(\boldsymbol{\sigma}, \mathbf{B})=\frac{1}{p_{r}{ }^{1-n} k(1-n)(2-n)} \bar{p}_{0}^{2-n}= \\
& \quad=\frac{1}{p_{r}{ }^{1-n} k(1-n)(2-n)}\left[\left(\frac{1}{9}-\frac{k(1-n)}{6 g}\right) \operatorname{tr}^{2}\left(\mathbf{B}^{-1} \boldsymbol{\sigma}\right)+\frac{k(1-n)}{2 g} \operatorname{tr}\left[\left(\mathbf{B}^{-1} \boldsymbol{\sigma}^{s y m}\right)^{2}\right]\right]^{\frac{2-n}{2}}
\end{aligned}
$$

The strain tensor is obtained differentiating the complementary energy with respect to the stress:

$$
\boldsymbol{\varepsilon}=\frac{1}{2 p_{r}^{1-n} k(1-n)} \bar{p}_{0}^{-n}\left[2\left(\frac{1}{9}-\frac{k(1-n)}{6 g}\right) \operatorname{tr}\left(\mathbf{B}^{-1} \boldsymbol{\sigma}\right) \mathbf{B}^{-1}+\frac{k(1-n)}{g} \mathbf{B}^{-1} \boldsymbol{\sigma}^{s y m} \mathbf{B}^{-1}\right]
$$

and, with further differentiation, the compliance tensor is:

$$
\begin{aligned}
\mathbf{C} & =\frac{1}{p_{r}{ }^{1-n} k(1-n)}\left\{\left(-\frac{n}{2}\right) \bar{p}_{0}^{-(n+2)} 2\left(\frac{1}{9}-\frac{k(1-n)}{6 g}\right) \operatorname{tr}^{2}\left(\mathbf{B}^{-1} \boldsymbol{\sigma}\right)+\right. \\
& \left.+\bar{p}_{0}^{-n}\left(\frac{1}{9}-\frac{k(1-n)}{6 g}\right)\right\} \mathbf{B}^{-1} \otimes \mathbf{B}^{-1}+ \\
& -\frac{n}{2 p_{r}{ }^{1-n} g} \bar{p}_{0}^{-(n+2)}\left(\frac{1}{9}-\frac{k(1-n)}{6 g}\right) \operatorname{tr}\left(\mathbf{B}^{-1} \boldsymbol{\sigma}\right)\left(\mathbf{B}^{-1} \otimes \mathbf{B}^{-1} \boldsymbol{\sigma}^{s y m} \mathbf{B}^{-1}+\mathbf{B}^{-1} \boldsymbol{\sigma}^{s y m} \mathbf{B}^{-1} \otimes \mathbf{B}^{-1}\right)+ \\
& -\frac{n}{4 p_{r}{ }^{1-n}} \bar{p}_{0}^{-(n+2)} \frac{k(1-n)}{g^{2}}\left(\mathbf{B}^{-1} \boldsymbol{\sigma}^{s y m} \mathbf{B}^{-1} \otimes \mathbf{B}^{-1} \boldsymbol{\sigma}^{s y m} \mathbf{B}^{-1}\right)+\frac{1}{2 p_{r}{ }^{1-n} g} \bar{p}_{0}^{-n} \mathbf{B}^{-1} \otimes \mathbf{B}^{-1}
\end{aligned}
$$

The strain energy function of the proposed nonlinear anisotropic hyperelastic model expressed in terms of the isotropic and deviatoric parts of $\mathbf{B}$ takes the form:

$$
\begin{aligned}
& E(\boldsymbol{\varepsilon}, f, \mathbf{F})=\frac{p_{r}}{k(2-n)} \bar{r}_{0}^{\frac{2-n}{1-n}}=\frac{p_{r}}{k(2-n)} k^{\frac{2-n}{2-2 n}}(1-n)^{\frac{2-n}{2-2 n}} \\
& \left\{\left[k(1-n)-\frac{2}{3} g\right][\operatorname{tr}(\boldsymbol{\varepsilon}) f+\operatorname{tr}(\mathbf{F} \boldsymbol{\varepsilon})]^{2}+2 g \operatorname{tr}\left(f \boldsymbol{\varepsilon}^{s y m}+\mathbf{F} \boldsymbol{\varepsilon}^{s y m}\right)^{2}\right\}^{\frac{2-n}{2-2 n}}
\end{aligned}
$$

Differentiating eq. (A10) with respect to the strains, one obtains the stress tensor

$$
\begin{aligned}
\boldsymbol{\sigma} & =p_{r} \bar{r}_{0}^{\frac{n}{1-n}}\left\{\left[k(1-n)-\frac{2}{3} g\right] \operatorname{tr}(f \boldsymbol{\varepsilon}+\mathbf{F} \boldsymbol{\varepsilon})(f \mathbf{I}+\mathbf{F})+\right. \\
& \left.+2 g\left(f^{2} \boldsymbol{\varepsilon}^{\text {sym }}+f \boldsymbol{\varepsilon}^{s y m} \mathbf{F}+f \mathbf{F} \boldsymbol{\varepsilon}^{s y m}+\mathbf{F} \boldsymbol{\varepsilon}^{s y m} \mathbf{F}\right)\right\}
\end{aligned}
$$


and, with a further differentiation, the stiffness tensor:

$$
\begin{aligned}
& \mathbf{D}=p_{r}\left[k(1-n)-\frac{2}{3} g\right]\left\{k \bar{r}_{0}^{\frac{3 n-2}{1-n}} n\left[k(1-n)-\frac{2}{3} g\right] \operatorname{tr}^{2}(f \boldsymbol{\varepsilon}+\mathbf{F} \boldsymbol{\varepsilon})+\bar{r}_{0}^{\frac{n}{1-n}}\right\} \\
& \left(f^{2} \mathbf{I} \otimes \mathbf{I}+f \mathbf{I} \otimes \mathbf{F}+f \mathbf{F} \otimes \mathbf{I}+\mathbf{F} \otimes \mathbf{F}\right)+ \\
& +4 p_{r} k \bar{r}_{0}^{\frac{3 n-2}{1-n}} n g^{2}\left(f^{4} \boldsymbol{\varepsilon}^{s y m} \otimes \boldsymbol{\varepsilon}^{s y m}+f^{3} \boldsymbol{\varepsilon}^{s y m} \otimes \boldsymbol{\varepsilon}^{s y m} \mathbf{F}+f^{2} \boldsymbol{\varepsilon}^{s y m} \otimes \mathbf{F} \boldsymbol{\varepsilon}^{s y m} \mathbf{F}+f^{3} \boldsymbol{\varepsilon}^{s y m} \otimes \mathbf{F} \boldsymbol{\varepsilon}^{s y m}+\right. \\
& +f^{3} \boldsymbol{\varepsilon}^{s y m} \mathbf{F} \otimes \boldsymbol{\varepsilon}^{s y m}+f^{2} \boldsymbol{\varepsilon}^{s y m} \mathbf{F} \otimes \boldsymbol{\varepsilon}^{s y m} \mathbf{F}+f \boldsymbol{\varepsilon}^{s y m} \mathbf{F} \otimes \mathbf{F} \boldsymbol{\varepsilon}^{s y m} \mathbf{F}+f^{2} \boldsymbol{\varepsilon}^{s y m} \mathbf{F} \otimes \mathbf{F} \boldsymbol{\varepsilon}^{s y m}+f^{2} \mathbf{F} \boldsymbol{\varepsilon}^{s y m} \mathbf{F} \otimes \boldsymbol{\varepsilon}^{s y m}+ \\
& +f \mathbf{F} \boldsymbol{\varepsilon}^{s y m} \mathbf{F} \otimes \boldsymbol{\varepsilon}^{s y m} \mathbf{F}++\mathbf{F} \boldsymbol{\varepsilon}^{s y m} \mathbf{F} \otimes \mathbf{F} \boldsymbol{\varepsilon}^{s y m} \mathbf{F}+f \mathbf{F} \boldsymbol{\varepsilon}^{s y m} \mathbf{F} \otimes \mathbf{F} \boldsymbol{\varepsilon}^{s y m}+f^{3} \mathbf{F} \boldsymbol{\varepsilon}^{s y m} \otimes \boldsymbol{\varepsilon}^{s y m}+f^{2} \mathbf{F} \boldsymbol{\varepsilon}^{\text {sym }} \otimes \boldsymbol{\varepsilon}^{s y m} \mathbf{F}+ \\
& \left.+f \mathbf{F} \boldsymbol{\varepsilon}^{s y m} \otimes \mathbf{F} \boldsymbol{\varepsilon}^{\text {sym }} \mathbf{F}+f^{2} \mathbf{F} \boldsymbol{\varepsilon}^{s y m} \otimes \mathbf{F} \boldsymbol{\varepsilon}^{\text {sym }}\right) \\
& +2 p_{r} k \bar{r}_{0}^{\frac{3 n-2}{1-n}} n g\left[k(1-n)-\frac{2}{3} g\right] \operatorname{tr}(f \boldsymbol{\varepsilon}+\mathbf{F} \boldsymbol{\varepsilon})\left(f^{3} \mathbf{I} \otimes \boldsymbol{\varepsilon}^{s y m}+f^{2} \mathbf{I} \otimes \boldsymbol{\varepsilon}^{s y m} \mathbf{F}+f \mathbf{I} \otimes \mathbf{F} \boldsymbol{\varepsilon}^{s y m} \mathbf{F}+f^{2} \mathbf{I} \otimes \mathbf{F} \boldsymbol{\varepsilon}^{s y m}+\right. \\
& +f^{2} \mathbf{F} \otimes \boldsymbol{\varepsilon}^{s y m}+f \mathbf{F} \otimes \boldsymbol{\varepsilon}^{s y m} \mathbf{F}+\mathbf{F} \otimes \mathbf{F} \boldsymbol{\varepsilon}^{s y m} \mathbf{F}+f \mathbf{F} \otimes \mathbf{F} \boldsymbol{\varepsilon}^{s y m}+f^{3} \boldsymbol{\varepsilon}^{s y m} \otimes \mathbf{I}+f^{2} \boldsymbol{\varepsilon}^{s y m} \mathbf{F} \otimes \mathbf{I}+f \mathbf{F} \boldsymbol{\varepsilon}^{s y m} \mathbf{F} \otimes \mathbf{I}+ \\
& \left.+f^{2} \mathbf{F} \boldsymbol{\varepsilon}^{\text {sym }} \otimes \mathbf{I}+f^{2} \boldsymbol{\varepsilon}^{\text {sym }} \otimes \mathbf{F}+f \boldsymbol{\varepsilon}^{s y m} \mathbf{F} \otimes \mathbf{F}+\mathbf{F} \boldsymbol{\varepsilon}^{\text {sym }} \mathbf{F} \otimes \mathbf{F} \boldsymbol{\varepsilon}^{\text {sym }} \mathbf{F}+f \mathbf{F} \boldsymbol{\varepsilon}^{\text {sym }} \otimes \mathbf{F}\right)+ \\
& +2 p_{r} \bar{r}^{\frac{n}{1-n}} g\left(f^{2} \mathbf{I} \underline{\otimes} \mathbf{I}+f \mathbf{I} \underline{\otimes} \mathbf{F}+f \mathbf{F} \underline{\otimes} \mathbf{I}+\mathbf{F} \underline{\otimes} \mathbf{F}\right)
\end{aligned}
$$


List of Tables

\begin{tabular}{|c|c|}
\hline$p_{r}$ & 100 \\
\hline$n$ & 0.47 \\
\hline$k$ & 1250 \\
\hline$g$ & 1050 \\
\hline
\end{tabular}

Table 1 - Model parameters

\begin{tabular}{|c|c|}
\hline$\varepsilon_{11}$ & 0.001 \\
\hline$\varepsilon_{22}$ & 0.001 \\
\hline$\varepsilon_{33}$ & 0.001 \\
\hline$\varepsilon_{12}$ & 0 \\
\hline$\varepsilon_{13}$ & 0 \\
\hline$\varepsilon_{23}$ & 0 \\
\hline
\end{tabular}

\begin{tabular}{|c|c|}
\hline$\sigma_{11}$ & 365 \\
\hline$\sigma_{22}$ & 365 \\
\hline$\sigma_{33}$ & 365 \\
\hline$\sigma_{12}$ & 0 \\
\hline$\sigma_{13}$ & 0 \\
\hline$\sigma_{23}$ & 0 \\
\hline
\end{tabular}

\begin{tabular}{|c|c|c|c|c|c|}
\hline 487284 & 101134 & 101134 & 0 & 0 & 0 \\
\hline 101134 & 487284 & 101134 & 0 & 0 & 0 \\
\hline 101134 & 101134 & 487284 & 0 & 0 & 0 \\
\hline 0 & 0 & 0 & 193075 & 0 & 0 \\
\hline 0 & 0 & 0 & 0 & 193075 & 0 \\
\hline 0 & 0 & 0 & 0 & 0 & 193075 \\
\hline
\end{tabular}

Table 2 - Strain, stress and stiffness tensors (isotropic strain state), stress and stiffness are in $\mathrm{kPa}$

\begin{tabular}{|c|c|}
\hline$\varepsilon_{11}$ & 0.0015 \\
\hline$\varepsilon_{22}$ & 0.001 \\
\hline$\varepsilon_{33}$ & 0.001 \\
\hline$\varepsilon_{12}$ & 0 \\
\hline$\varepsilon_{13}$ & 0 \\
\hline$\varepsilon_{23}$ & 0 \\
\hline
\end{tabular}

\begin{tabular}{|c|c|}
\hline$\sigma_{11}$ & 648 \\
\hline$\sigma_{22}$ & 423 \\
\hline$\sigma_{33}$ & 423 \\
\hline$\sigma_{12}$ & 0 \\
\hline$\sigma_{13}$ & 0 \\
\hline$\sigma_{23}$ & 0 \\
\hline
\end{tabular}

\begin{tabular}{|c|c|c|c|c|c|}
\hline 648072 & 125671 & 125671 & 0 & 0 & 0 \\
\hline 125671 & 530238 & 79157 & 0 & 0 & 0 \\
\hline 125671 & 79157 & 530238 & 0 & 0 & 0 \\
\hline 0 & 0 & 0 & 225540 & 0 & 0 \\
\hline 0 & 0 & 0 & 0 & 225540 & 0 \\
\hline 0 & 0 & 0 & 0 & 0 & 225540 \\
\hline
\end{tabular}

Table 3 - Strain, stress and stiffness tensors (anisotropic strain state)

\begin{tabular}{|c|c|}
\hline$\varepsilon_{11}$ & 0.001 \\
\hline$\varepsilon_{22}$ & 0.001 \\
\hline$\varepsilon_{33}$ & 0.001 \\
\hline$\varepsilon_{12}$ & 0.001 \\
\hline$\varepsilon_{13}$ & 0 \\
\hline$\varepsilon_{23}$ & 0 \\
\hline
\end{tabular}

\begin{tabular}{|c|c|}
\hline$\sigma_{11}$ & 463 \\
\hline$\sigma_{22}$ & 463 \\
\hline$\sigma_{33}$ & 463 \\
\hline$\sigma_{12}$ & 489 \\
\hline$\sigma_{13}$ & 0 \\
\hline$\sigma_{23}$ & 0 \\
\hline
\end{tabular}

\begin{tabular}{|c|c|c|c|c|c|}
\hline 560694 & 71553 & 71553 & 84832 & 0 & 0 \\
\hline 71553 & 560694 & 71553 & 84832 & 0 & 0 \\
\hline 71553 & 71553 & 560694 & 84832 & 0 & 0 \\
\hline 84832 & 84832 & 84832 & 334205 & 0 & 0 \\
\hline 0 & 0 & 0 & 0 & 244570 & 0 \\
\hline 0 & 0 & 0 & 0 & 0 & 244570 \\
\hline
\end{tabular}

Table 4 - Strain, stress and stiffness tensors (in presence of shear strain)

\begin{tabular}{|c|c|c|c|}
\hline & $\begin{array}{c}\text { Ham River } \\
\text { Sand }\end{array}$ & Ticino River Sand & London Clay \\
\hline$p_{r}(\mathrm{kPa})$ & 100 & 100 & 100 \\
\hline$n$ & 0.47 & 0.5 & 0.8 \\
\hline$k$ & 1250 & 1300 & 350 \\
\hline$g$ & 1050 & 940 & 340 \\
\hline$a_{22} / a_{11}$ & 0.922 & $\begin{array}{c}\text { Varies as a function of } K_{o} \\
0.98-1.118\end{array}$ & 1.378 \\
\hline
\end{tabular}

Table 5: parameters for various soils 


\section{Figure captions}

Fig. 1 Constant volumetric (continuous line) and constant deviatoric (dashed lines) strain contours.

Fig. 2 Evolution of Poisson's ratio during isotropic consolidation (modified from Ezaoui and Di Benedetto [15])

Fig. 3 Effective anisotropic stress path (modified from Kuwano and Jardine [32])

Fig. 4 Ham River sand: evolution of Young's moduli (a) and shear moduli (b) during anisotropic consolidation

Fig. 5 Ticino River sand: evolution of Young's moduli (a) and shear moduli (b) during anisotropic consolidation $-K_{0}=0.5$

Fig. 6 Ticino River sand: evolution of Young's moduli (a) and shear moduli (b) during isotropic consolidation $-K_{0}=1$

Fig. 7 Ticino River sand: evolution of Young's moduli (a) and shear moduli (b) during anisotropic consolidation $-K_{0}=1.5$

Fig. 8 Stress states reconsolidation of London clay samples (modified from Hight et al. [22])

Fig. 9 Consolidation paths of London clay samples (modified from Gasparre et al. [19])

Fig. 10 London Clay: evolution of Young's moduli (a) and shear moduli (b) with mean effective pressure

Fig. 11 London Clay: evolution of shear moduli during isotropic consolidation

\section{Notation}

$\begin{array}{ll}\mathbf{a}, a_{i j} & \text { Anisotropy tensor } \\ \mathbf{B}, B_{i j} & \text { Anisotropy tensor } \mathbf{B}=\mathbf{a}^{2}, B_{i j}=a_{i k} a_{k j} \\ c_{1}-c_{9} & \text { Coefficients in general anisotropy expression } \\ C & \text { Complementary energy } \\ C_{i j} & \text { Compliance tensor in triaxial formulation } \\ \mathrm{C}, C_{i j k l} & \text { Compliance tensor } \\ \mathrm{D}, D_{i j k l} & \text { Stiffness tensor } \\ E & \text { Strain energy } \\ E_{h} & \text { Horizontal Young's modulus } \\ E_{v} & \text { Vertical Young's modulus } \\ f & \text { Constant of eq. (11) } \\ \mathbf{F}, F_{i j} & \text { Deviatoric part of the fabric tensor } \\ g & \text { Dimensionless shear modulus coefficient } \\ G & \text { Shear modulus } \\ G_{0}^{r e f} & \text { Reference shear modulus of eq. (44) } \\ G_{h h} & \text { Shear modulus in the horizontal plane } \\ G_{h v}, G_{v h} & \text { Shear modulus in vertical planes }\end{array}$




\begin{tabular}{|c|c|}
\hline I & Identity tensor \\
\hline$k$ & Dimensionless bulk modulus coefficient \\
\hline$K$ & Bulk modulus \\
\hline$K_{0}$ & Coefficient of earth pressure at rest \\
\hline$l$ & Exponent in eq. (48) \\
\hline $\mathbf{M}_{i}$ & Eigenprojections of the fabric tensor \\
\hline$n$ & (1) Exponent in power-law relationship for elastic stiffness, (2) exponent in eq. (48) \\
\hline$n$ & Vector of internal structure orientation \\
\hline$N$ & Switch parameter \\
\hline $\mathbf{N}$ & Fabric tensor of eq. (44) \\
\hline$p$ & Mean effective stress \\
\hline $\mathbf{p}$ & Fabric tensor of eq. (34) \\
\hline $\bar{p}_{0}$ & Stress function used in definition of nonlinearity \\
\hline$p_{r}$ & Reference pressure \\
\hline$p_{\text {ref }}$ & Reference pressure of eq. (44) \\
\hline$q$ & Deviatoric stress \\
\hline $\boldsymbol{q}_{i}$ & Orthonormal eigenvectors \\
\hline$\overline{r_{0}}$ & Strain function used in definition of nonlinearity \\
\hline$v$ & Unit vector of Cudny and Partyka model [11] \\
\hline$y$ & Coefficient of eq. (30) \\
\hline$\alpha$ & (1) Coefficient of eq. (28), (2) parameter of eq. (48) \\
\hline$\beta$ & (1) Constant of eq. (44), (2) constant of eq. (48) \\
\hline$\delta_{i j}$ & Kronecker delta \\
\hline $\boldsymbol{\varepsilon}, \varepsilon_{i j}$ & Strain tensor \\
\hline$\overline{\boldsymbol{\varepsilon}}, \bar{\varepsilon}_{i j}$ & Equivalent symmetric strain tensor in anisotropic model \\
\hline $\boldsymbol{\varepsilon}^{\prime}, \varepsilon_{i j}^{\prime}$ & Deviatoric part of the strain tensor \\
\hline$\varepsilon_{s}$ & Deviatoric strain invariant \\
\hline$\varepsilon_{v}$ & Volumetric strain \\
\hline$\lambda$ & Lamé parameter \\
\hline$\mu$ & Lamé parameter \\
\hline$v$ & Poisson's ratio \\
\hline$\sigma, \sigma_{i j}$ & Stress tensor \\
\hline$\sigma^{\prime}, \sigma_{i j}^{\prime}$ & Deviatoric part of the stress tensor \\
\hline$\omega_{1}, \omega_{2}$ & Constants of eq. (32) \\
\hline
\end{tabular}

\section{References}

[1] Amorosi A, Boldini D, Germano V (2008) A generalised backward euler scheme for the integration of a mixed isotropic-kinematic hardening model for clays. International Journal of Numerical and Analytical Methods in Geomechanics 32: 1173-1203 
[2] Bellotti R, Jamiolkowski M, Lo Presti DCF, O’Neill DA (1996) Anisotropy of small strain stiffness in Ticino sand. Géotechnique 46(1): 115-131

[3] Bigoni D, Loret B (1999) Effects of elastic anisotropy on strain localization and flutter instability in plastic solids. Journal of the Mechanics and Physics of Solids 47(7): 1409-1436

[4] Boehler JP (1987) Applications of tensor functions in solid mechanics (Vol. 292). JP Boehler (Ed.). Springer, New York

[5] Boyce HR (1980) A nonlinear model for the elastic behaviour of granular materials under repeated loading. In Proc. Int. Symp. Soils under Cyclic and Transient Loading, Swansea, Vol. 1, pp. 285-294. Balkema Publ.

[6] Borja RI, Tamagnini C, Amorosi A (1997) Coupling plasticity and energy-conserving elasticity models for clays. ASCE, Journal of the Geotechnical and Geoenviromental Engineering Division 123(10): 948-957

[7] Chaudhary S, Kuwano J, Hayano Y (2004) Measurement of Quasi-Elastic Stiffness Parameters of Dense Toyoura Sand in Hollow Cylinder Apparatus and Triaxial Apparatus with Bender Elements. Geotechnical Testing Journal 27(1): 23-35

[8] Clayton CRI (2011) Stiffness at small strain: research and practice. Géotechnique 61(1): 5-37.

[9] Collins IF, Houlsby GT (1997) Application of thermomechanical principles to the modelling of geotechnical materials. Proc. of the Royal Society of London A: Mathematical, physical and engineering sciences (Vol. 453, No. 1964, pp. 19752001)

[10] Cowin SC (1985) The relationship between the elasticity tensor and the fabric tensor. Mechanics of Materials 4(2): $137-147$

[11] Cudny M, Partyka E (2017) Influence of anisotropic stiffness in numerical analyses of tunneling and excavation problems in stiff soils. In Lee, Woojin, Lee, Jong-Sub, Kim, Hyun-Ki, \& Kim, Dong-Soo (Eds.) Proceedings of the 19th International Conference on Soil Mechanics and Geotechnical Engineering, Seoul 2017, International Society for Soil Mechanics and Geotechnical Engineering, Seoul, Korea, pp. 719-722

[12] Dafalias YF, Manzari MT (2004) Simple plasticity sand model accounting for fabric change effects. Journal of Engineering Mechanics 130(6): 622-634

[13] De Silva LIN (2004) Locally measured quasi-elastic deformation properties of geomaterials under torsional shear and triaxial loadings. Master's degree Dissertation, The University of Tokyo

[14] Einav I, Puzrin AM (2004) Pressure-dependent elasticity and energy conservation in elastoplastic models for soils. Journal of Geotechnical and Geoenvironmental Engineering 130(1): 81-92

[15] Ezaoui A, Benedetto HD (2009) Experimental measurements of the global anisotropic elastic behaviour of dry Hostun sand during triaxial tests, and effect of sample preparation. Géotechnique 59(7): 621-635

[16] Franzius JN, Potts DM, Burland JB (2005) The influence of soil anisotropy and K 0 on ground surface movements resulting from tunnel excavation. Géotechnique 55(3): 189-199

[17] Gajo A, Bigoni D (2008) A model for stress and plastic strain induced nonlinear, hyperelastic anisotropy in soils. International Journal for Numerical and Analytical Methods in Geomechanics 32(7): 833-861 
[18] Gasparre A (2005) Advanced laboratory characterisation of London Clay. PhD Thesis, Imperial College London

[19] Gasparre A, Nishimura S, Minh NA, Coop MR, Jardine RJ (2007) The stiffness of natural London Clay. Géotechnique 57(1): 33-47

[20] Giot R, Giraud A, Guillon T, Auvray C (2012) Three-dimensional poromechanical back analysis of the pulse test accounting for transverse isotropy. Acta Geotechnica 7(3): 151-165

[21] Graham J, Houlsby GT (1983) Anisotropic elasticity of a natural clay. Géotechnique 33(2): 165-180

[22] Hight DW, McMillan F, Powell JJM, Jardine RJ, Allenou CP (2003) Some characteristics of London clay. Characterisation and engineering properties of natural soils, 2 (eds T. S. Tan, K. K. Phoon, D. W. Hight and S. Leroueil) 851-946. Rotterdam: Balkema

[23] Hight DW, Gasparre A, Nishimura S, Minh NA, Jardine RJ, Coop MR (2007) Characteristics of the London Clay from the Terminal 5 site at Heathrow Airport. Géotechnique 57(1): 3-18

[24] HongNam N, Koseki J (2005) Quasi-elastic deformation properties of Toyoura sand in cyclic triaxial and torsional loadings. Soils and foundations 45(5): 19-37

[25] Houlsby GT (1985) The use of a variable shear modulus in elastic-plastic models for clays. Computers and Geotechnics 1(1): 3-13

[26] Houlsby GT, Amorosi A, Rojas E (2005) Elastic moduli of soils dependent on pressure: a hyperelastic formulation. Géotechnique 55(5): 383-392

[27] Houlsby GT, Amorosi A, Rollo F (2018) Non-linear anisotropic hyperelasticity for granular materials. Submitted for publication

[28] Ibraim E, Christiaens P, Pope M (2011) Development of a hollow cylinder torsional apparatus for pre-failure deformation and large strains behaviour of sand. Geotechnical Engineering Journal of the South-east Asian Geotechnical Society (SEAGS) \& Association of Geotechnical Societies in South-East Asia (AGSSEA), Special Issue on Soil Behaviour 42(4): 58-68. ISSN 0046-5828

[29] Itskov M (2007) Tensor Algebra and Tensor Analysis for Engineers. Springer.

[30] Jiang Y, Zheng H, Peng Z, Fu L, Song S, Sun Q, Liu M (2012) Expression for the granular elastic energy. Physical Review E 85(5): 051304

[31] Kuhn MR, Sun W, Wang Q (2015) Stress-induced anisotropy in granular materials: fabric, stiffness, and permeability. Acta Geotechnica 10(4): 399-419

[32] Kuwano R, Jardine RJ (2002) On the applicability of cross-anisotropic elasticity to granular materials at very small strains. Géotechnique 52(10): 727-749

[33] Lashkari A (2010) A SANISAND model with anisotropic elasticity. Soil Dynamics and Earthquake Engineering 30(12): 1462-1477

[34] Lodge AS (1955) The transformation to isotropic form of the equilibrium equations for a class of anisotropic elastic solids. Quarterly Journal of Mechanics and Applied Mathematics 8(2): 211-225 
[35] Maier G, Hueckel T (1979) Nonassociated and coupled flow rules of elastoplasticity for rock-like materials. International Journal of Rock Mechanics and Mining Sciences \& Geomechanics Abstracts 16(2): 77-92

[36] Mašín D, Rott J (2014) Small strain stiffness anisotropy of natural sedimentary clays: review and a model. Acta Geotechnica 9(2): 299-312

[37] Mital U, Kawamoto R, Andrade JE Effect of fabric on shear wave velocity in granular soils. Acta Geotechnica. https://doi.org/10.1007/s11440-019-00766-1

[38] Mitaritonna G, Amorosi A, Cotecchia F (2014) Experimental investigation of the evolution of elastic stiffness anisotropy in a clayey soil. Géotechnique 64(6): 463-475

[39] Niemunis A, Cudny M (1998) On hyperelasticity for clays. Computers and Geotechnics 23(4): 221-236

[40] Puzrin AM, Burland JB, Standing JR (2012) Simple approach to predicting ground displacements caused by tunnelling in undrained anisotropic elastic soil. Géotechnique 62(4): 341-352

[41] Tamagnini C, Castellanza R, Nova R (2002) A generalized backward Euler algorithm for the numerical integration of an isotropic hardening elastoplastic model for mechanical and chemical degradation of bonded geomaterials. International Journal of Analytical and Numerical Methods in Geomechanics 26: 963-1004

[42] Truesdell CA, Noll W (1965) Handbuch der physik. Springer

[43] Vermeer PA (1982) A five-constant model unifying well-established concepts. Gudehus, Darve and Vardoulakis (eds). Results of the International Workshop on Constitutive Relations for Soils, Grenoble, Balkema, 175-197

[44] Wang CC (1970) A new representation theorem for isotropic functions: An answer to Professor GF Smith's criticism of my papers on representations for isotropic functions. Archive for Rational Mechanics and Analysis 36(3): 166-197

[45] Xiao Y, Zhang Z, Wang J (2019) Granular hyperelasticity with inherent and stress-induced anisotropy. Acta Geotechnica. https://doi.org/10.1007/s11440-019-00768-Z

[46] Yang LT. Li X, Yu HS, Wanatowski D (2016) A laboratory study of anisotropic geomaterials incorporating recent micromechanical understanding. Acta Geotechnica 11(5): 1111-1129

[47] Zhao J, Gao Z (2015) Unified anisotropic elastoplastic model for sand. Journal of Engineering Mechanics 142(1): 04015056

[48] Zysset PK, Curnier A (1995) An alternative model for anisotropic elasticity based on fabric tensors. Mechanics of Materials 21(4): 243-250 